\title{
Identification and Pyramid of QTLs Based on Rice Short-wide Grain CSSL-Z414, SSSL, DSSL and Candidate Gene Analysis of qGL11 and qGW5
}

Juan Li

Southwest University

Hongxia Yang

Southwest University

Guangyi Xu

Southwest University

Keli Deng

Southwest University

Jinjin Yu

Southwest University

Siqian Xiang

Southwest University

Kai Zhou

Southwest University

Qiuli Zhang

Southwest University

Ruxiang Li

Southwest University

Miaomiao Li

Southwest University

Yinghua Ling

Southwest University

Zhenglin Yang

Southwest University

Guanghua He

Southwest University

Fangming Zhao ( $\nabla$ zhaofangming2004@163.com )

Southwest University https://orcid.org/0000-0003-2781-0452 
Keywords: Rice, Chromosome segment substitution line, Grain size, QTL, CycT1, 3

Posted Date: September 21st, 2021

DOI: https://doi.org/10.21203/rs.3.rs-889515/v1

License: (c) (1) This work is licensed under a Creative Commons Attribution 4.0 International License. Read Full License 


\section{Abstract}

\section{Background}

Most of rice agronomic traits as grain length etc. are complex traits controlled by multiple genes. Chromosome segment substitution lines (CSSLs) are ideal materials for dissecting and studying of these complex traits.

\section{Results}

A rice short-wide grain CSSL Z414 was identified among progeny of the recipient parent Xihui 18 (an indica restorer line) and the donor parent Huhan 3 (a japonica cultivar). Z414 carried 4 substitution segments (average length was $3.04 \mathrm{Mb}$ ), and displayed shorter panicle length and less number of primary branches, shorter, wider and larger grain, higher brown rice rate and chalkiness degree when compared with Xihui 18. Then, 9 quantitative trait loci (QTLs) for associated traits were identified using the secondary $\mathrm{F}_{2}$ population from Xihui 18 / Z414. Among them, 6 QTLs ( $q P L 3, q G W 5, q G L 11, q R L W 5$, qRLW11, qGWT5) could be verified by corresponding single segment substitution lines (SSSLs, S1-S6). In addition, 4 QTLs ( $q G L 3, q G L 5, q C D 3$ and $q C D 5$ ) were detected by $\mathrm{S} 1$ and $\mathrm{S} 5$, which was not detected by the $\mathrm{F}_{2}$ population. Thus, the grain length of $Z 414$ was controlled by $q G L 11, q G L 3$ and $q G L 5$, and the grain width of Z414 was answered by $q G W 5$. Then by substitution mapping, $q G L 11$ and $q G W 5$ were delimited within the estimated substitution length of 1.42 and $1.14 \mathrm{Mb}$ on chromosomes 11 and 5 , and 4 and 2 candidate genes were found respectively for $q G L 11$ and $q G W 5$ by sequencing. However, only two had expression differences by qRT-PCR analysis. Finally, Analysis of QTL epistatic effects revealed that pyramid of $q G L 3(a=0.22)$ and $q G L 11(a=-0.19)$ caused grain length of double segment substitution line (DSSL, D2) shorter than that of S5 ( $q G L 11)$.

\section{Conclusions}

We developed a rice short - wide grain CSSL with 4 substitution segments from Huhan 3 based on the genetic backgrounds of Xihui 18 . The grain width of Z414 was controlled by $q G W 5$, and GS5 should be the candidate gene for $q G W 5$ by sequencing and qRT-PCR analysis. The grain length of Z414 was controlled by $q G L 11, q G L 3$, and $q G L 5$, and $C y C T 1 ; 3$ should be the best candidate gene of $q G L 11$, whose specific function of regulating grain length was still unknown, and $q G L 11$ is epistatic to $q G L 3$.

\section{Background}

Rice (Oryza sativa L.) is one of the most important cereal crops in the world, providing staple food for more than half of the world's population ( $\mathrm{Li}$ et al. 2014). It is necessary to continue to improve rice breeding efficiency. At the beginning of the post-genomic era, a new concept of "breeding by design" was proposed, which aims to control all allelic variation for all genes of plant agronomic importance (Zhang et al. 2021a). The core of rice design breeding is the use of naturally occurring variation (Zhang et al. 2021a). First of all, identification of favorable alleles is principle. Here, a rice short -wide grain 
chromosome segment substitution line (CSSL) was identified from naturally occurring variation. To detect the alleles for grain size in the CSSL are important for our carrying out the plan of design breeding. Grain size and weight are important target traits determining grain yield and quality in rice (Feng et al, 2021).

At present, more than 400 QTLs related to grain size have been mapped (Huang et al. 2013). Some of them have been cloned. Several signaling pathways that determine grain size have been identified by these cloned genes, including phytohormones pathway, G protein signal pathway, ubiquitin-proteasome pathway, mitogen-activated protein kinase (MAPK) signal transduction pathway, and transcription factor regulation (Li et al. 2016). Several phytohormones such as brassinosteroids (BRs) and auxins (IAA) have been suggested to play an important role in rice grain size. In rice, Three QTLs (GS5, qGL3/GL3.1 and GW5) for grain size might be involved in BR signaling. GS5 encodes a putative serine carboxypeptidase, and competitively inhibits the interaction between OsBAK1-7 and OsMSBP1 by influencing BR signaling. Higher expression of GS5 results in wide and heavy grains as a result of increased cell proliferation and expansion in spikelet hulls (Li et al. 2011; Xu et al. 2015a). GL3.1 suppresses BR signaling by regulating the phosphorylation and stability of OsGSK3 (Gao et al. 2019). GW5 can repress the kinase activity of GSK2, resulting in accumulation of unphosphorylated OSBZR1 and $D L T$ proteins in the nucleus to mediate BR responsive gene expression and growth responses, thus affect the grain width (Liu et al. 2017). Two QTLs (TGW6 and BG1) for rice grain size might participate in IAA signaling. TGW6 encodes protein with indole-3-acetic acid (IAA)-glucose hydrolase activity. In sink organs, the Nipponbare tgw6 allele limits cell number and grain length by controlling IAA supply. Loss of function of the Kasalath allele enhances grain weight and yield significantly (Ishimaru et al. 2013). BG1 controls rice grain size as a positive regulator of IAA response and transport (Liu et al. 2015a). G protein signaling is involved in regulating grain size in rice. In the pathway, heterotrimeric $\mathrm{G}$ protein is usually composed of five subunits. Ga subunits of $R G A 1$ provides a foundation for grain size expansion, $\mathrm{G} \beta$ subunits of $R G B 1$ is essential for plant survival and growth, Gy subunits of GS3 act as a brake in this pathway, GS3 reduces grain length by competing for binding to RGB1 to inhibits the downstream signal of DEP1/GGC2 (Sun et al. 2018). Ubiquitin can directly or indirectly regulate grain size by affecting protein transport, signal transduction and degradation. GW2 encoding a RING-type protein with E3 ubiquitin ligase activity negatively regulates cell division by targeting its substrate to proteasomes for regulated proteolysis. $1 \mathrm{bp}$ missing in the GW2 allele from WY3 causes the terminate translation prematurely during the transcription process, which makes the substrates that should be degraded cannot be recognition especially, and the grain length becomes longer (Song et al. 2007). LG1 encodes OsUBP15 that possesses de-ubiquitination activity in vitro, loss-of-function and down-regulated expression of OsUBP15 produced smaller grains (Shi et al. 2019). MAPK cascades transmit developmental signals to target molecules through sequential phosphorylation (Xu et al. 2015b). OsMKKK10, OsMKK4 and OsMAPK6 act in the same MAPK pathway to regulates grain size as a positive regulator (Xu et al. 2018). GSN1 is a negative regulator of the OsMKKK10-OsMKK4-OsMPK6 cascade, gsn1 mutant has larger grain size (Guo et al. 2018). Transcription regulators are considered to be important factors in controlling plant seed size. GS2 affects grain size by encoding a transcription factor OsGRF4 (Duan et al. 2015). GW8 is an SBP-domain transcription factor, 
regulates grain width as a positive regulator, can bound directly to the GW7 promoter and repressed its expression (Wang et al. 2015).

Although several genes for rice grain size have been cloned, it is still fragmentary for our understanding of the underlying mechanisms that regulate rice grain size. Thus, it is necessary to identify more QTLs for grain size. Here, we identified a rice short-wide grain chromosome segment substitution line (CSSL) Z414 with 4 substitution segments, derived from indica restorer line Xihui 18 as recipient parent and Huhan 3 as donor parent. We characterized Z414 genetically and mapped quantitative trait loci (QTLs) for associated traits by a secondary $F_{2}$ population derived from a cross between Xihui18 and Z414. In addition, we developed single-segment (SSSLs) and double-segment (DSSLs) for each QTL in the $F_{3}$ generation using marker-assisted selection (MAS). We verified the accuracy of QTL mapping using SSSLs. We conducted analysis of the additive and epistatic effects of QTLS on associated traits and assessed the effect of QTL pyramiding using DSSLs. In addition, the major QTLs qGL11 and qGW5 were substitution-mapped and candidate genes were analyzed by DNA sequencing and qRT-PCR .

\section{Results}

\section{Identification of substitution segments of Z414}

Based on the development of Z414, 8 polymorphic SSR markers on the substitution segments and 24 SSR markers outside the substitution segments of Z414 were used for further identification of substitution segments and purity detection of its genetic backgrounds using 10 plants of Z414. The results showed that the substitution segments of 10 plants of Z414 were consistent and no other residual segments from Huhan 3 were detected. Z414 contained 4 substitution segments from Huhan 3, which were distributed on the chromosome 3,5 and 11. The total estimated length of substitution segments was $12.17 \mathrm{Mb}$, and the average length was $3.04 \mathrm{Mb}$ (Fig. 1).

\section{Important traits assessment of Z414}

Compared with Xihui 18, Z414 displayed significant increase in grain width, 1000-grain weight, brown rice rate and chalkiness degree by $23.5 \%, 16.4 \%, 8.8 \%$ and $26.1 \%$, respectively. While there was significant decrease in panicle length, number of primary branches, grain length and ratio of length to width of Z414, reducing by $17.5 \%, 17.7 \%, 7.7 \%$ and $25.1 \%$, respectively. There were no significant differences in other traits, such as plant height, panicle number, number of secondary branches, spikelet number per panicle, grain number per panicle, seed setting rate, yield per plant, head rice rate, chalky rice rate and gel consistency (Fig. 2, Table 1).

\section{Cytological analysis of the glumes in Z414 and Xihui 18}

Scanning electron microscopy was used to analyze the cell morphology of glumes in Xihui 18 and Z414 at the heading stage. The cell width in the inner epidermis of the glumes of Z414 was increased significantly than that of Xihui 18 by $22.23 \%$. The total cell number in the outer epidermis of the glume 
along the longitudinal axis of Z414 was reduced significantly than that of Xihui 18 by $13.52 \%$ and the cell length in the inner epidermis of the glumes of Z414 exhibited no significant difference compared with that of Xihui 18 (Fig. 3). The results indicated that the short-wide grain of Z414 was mainly caused by the decrease of glume cell number and increase of glume cell width.

\section{Identification of QTL using the secondary $F_{2}$ population from Xihui18/Z414}

A total of 9 QTLs were detected on 3 substitution segments of Z414. They explained the phenotypic variation from $3.86 \%$ to $50.39 \%$ (Table 2). The allele qGW5 from Huhan 3 increased the grain width of Z414 by $0.12 \mathrm{~mm}$, explaining $50.39 \%$ of the phenotypic variation. The additive effect of $q G W T 5$ from Huhan 3 increased 1000-grain weight of Z414 by $0.63 \mathrm{~g}$. Furthermore, qGW5, qRLW5 and qGWT5 all linked to the same marker RM5874, suggesting that they should belong to pleiotropy. The allele $q G L 11$ from Huhan 3 reduced the grain length of $Z 414$ by $0.08 \mathrm{~mm}$, explaining $9.96 \%$ of the variation in grain length. Similarly, $q G L 11, q P L 11, q R L W 11, q G W T 11$ and $q B R R 11$ all linked to the same marker RM1812. However, the additive effects of $q R L W 11$ and $q G W T 11$ decreased the values of corresponding traits, while qPL 11 and $q B R R 11$ increased ones (Fig. 1, Table 2), indicating that these QTLs maybe belong to pleiotropy or cluster distribution. In addition, The additive effect of $q P L 3$ reduced panicle length of Z414 by $0.58 \mathrm{~cm}$ per panicle.

\section{Verification and pyramid of QTLs using the developed SSSLs and DSSLS}

On the basis of primary QTL mapping, 6 single segment substitution lines (SSSLs, S1-S6) and 2 double segment substitution lines (DSSLs, D1, D2) were developed in $\mathrm{F}_{3}$ by molecular marker assisted selection (MAS) method. Among them, S3, S4, and S5 belonged to SSSLs with overlapping substitution segments (Fig. 4 a).

6 QTLs ( $q P L 3, q G W 5, q G L 11, q R L W 5, q R L W 11, q G W T 5$ ) could be verified by SSSLs (Fig. 4a-f), which indicated that these QTLs could be inherited stably. 3 QTLs ( $q P L 11, q G W T 11$, and qBRR11) were not validated by 56 , suggesting that the expression of some minor QTLs might be easily influenced by the environment, whose contribution rates to phenotypic variation were only $7.33 \%$ for $\mathrm{qPL} 11,3.86 \%$ for qGWT11 and 5.92\% for qBRR11. In addition, 4 QTLs ( $q G L 3, q G L 5, q C D 3$ and $q C D 5$ ) for grain length and chalkiness degree were detected by $\mathrm{S} 1$ and $\mathrm{S} 5$, which were not detected in the secondary $\mathrm{F}_{2}$ population (Fig. 4c, g). The results showed that SSSLs had a higher efficiency of QTL detection.

The Panicle length $(25.49 \mathrm{~cm})$ of $\mathrm{S} 1$ carrying $q P L 3(\mathrm{a}=-0.77)$ was significantly shorter than that $(27.03 \mathrm{~cm})$ of Xihui 18 , while panicle length $(27.28,27.51,27.57,27.42$ and $26.45 \mathrm{~cm})$ of S2, S3, S4, S5, S6 without QTL for panicle length displayed no significant differences with that $(27.03 \mathrm{~cm})$ of Xihui 18 (Fig. 4a, b). Grain length ( 9.66 and $9.55 \mathrm{~mm}$ ) of S5 and S6 carrying QTLs ( $q G L 5$ and $q G L 11$ ) with negative effects were significantly shorter than that $(9.92 \mathrm{~mm})$ of Xihui 18 , while grain length $(10.35 \mathrm{~mm})$ of S1 carrying q GL3 with $0.22 \mathrm{~mm}$ of additive effect was significantly longer than that $(9.92 \mathrm{~mm})$ of Xihui 18 , the grain length $(9.85,9.92$ and $9.88 \mathrm{~mm})$ of S2, S3 and S4 without QTL for grain length exhibited no significant differences with that $(9.92 \mathrm{~mm}$ ) of Xihui 18 (Fig. 4a, c). Grain width (3.59 and $3.52 \mathrm{~mm}$ ) of S4 
and S5 harboring $q G W 5$ with positive additive effects were significantly wider than that $(3.12 \mathrm{~mm})$ of Xihui 18 , while grain width $(3.22,3.15,3.08$ and $3.20 \mathrm{~mm})$ of S1, S2, S3 and S6 without QTL for grain width showed no significant differences with that $(3.12 \mathrm{~mm})$ of Xihui 18 . The results indicated that $q G W 5$ was located in the substitution interval of RM405-RM5874-RM3322-RM3328 of chromosome 5 by theory of substitution mapping (Fig. 4a, d). 1000-grain weight (34.40 and $33.43 \mathrm{~g}$ ) of S4 and S5 containing $q G W T 5$ with positive effects were significantly larger than that $(30.75 \mathrm{~g})$ of Xihui 18 , and 1000 grain weight $(32.66,30.22,31.19$ and $31.98 \mathrm{~g})$ of S1, S2, S3, S6 without QTL for grain weight had no significant differences with that $(30.75 \mathrm{~g})$ of Xihui 18 . Similarly, $q G W T 5$ should be located in the substitution interval of RM405-RM5874-RM3322-RM3328 of chromosome 5 by theory of substitution mapping (Fig. 4a, e). Ratio of length to width (2.75, 2.75 and 2.99) of S4, S5 and S6 carrying QTLs with negative additive effects were significantly less than that (3.19) of Xihui 18, and ratio of length to width (3.22, 3.13 and 3.22) of S1, S2 and S3 without QTL for the trait displayed no significant differences with that (3.19) of Xihui 18. In the same way, qRLW5 should be located in the substitution interval of RM405RM5874-RM3322-RM3328 of chromosome 5(Fig. 4a, f). Chalkiness degree (19.76\%) of S1 harboring qCD3 $(\mathrm{a}=-0.59)$ was significantly lower than that of Xihui18 $(20.93 \%)$, while chalkiness degree $(24.02 \%)$ of 55 carrying $q C D 5(\mathrm{a}=1.54)$ was significantly higher than that $(20.93 \%)$ of Xihui 18 , the chalkiness degree $(20.62 \%, 22.00 \%, 22.35 \%$ and $22.35 \%)$ of S2, S3, S4 and S6 without QTL for chalkiness degree displayed no significant differences with that (20.93\%) of Xihui 18 (Fig. 4 g).

Pyramid of $q G L 3(\mathrm{a}=0.22)$ and $q G L 11(\mathrm{a}=-0.19)$ yielded an epistatic effect of -0.31 , which resulted in reduction $0.28 \mathrm{~mm}$ of the grain length in D2. The result suggested that pyramid of $q G L 3$ and $q G L 11$ resulted in shorter grains than $S 6$ (containing qGL 11) (Fig. 4c), indicating that $q G L 11$ displayed epistatic to $q G L 3$. Pyramid of $q G L 3(\mathrm{a}=0.22)$ and a substitution segment without QTL for grain length on chromosome 3 produced no epistatic effect in D1, which was consistent with the D1 phenotype (10.25 $\mathrm{mm})$, displaying no significant difference with that $(10.35 \mathrm{~mm})$ of $\mathrm{S} 1$, and significantly longer than that of Xihui18 and S2 (Fig. 4a, c). Pyramid the substitution segment without QTL for 1000-grain weight on chromosome 3 and another substitution segment on chromosome 11 yielded an epistatic effect of -2.94, resulting in decrease $2.94 \mathrm{~g}$ of 1000-grain weight genetically in D2, which matched with the phenotype $(28.99 \mathrm{~g})$ of D2, exhibiting significantly lower than that $(32.65,31.98$, and $30.75 \mathrm{~g})$ of S1, S6 and Xihui18 (Fig. 4a, e). The results suggested that pyramid of two substitution segments without QTL for grain weight (D2) yielded smaller 1000-grain weight than S1 and S6. In D1 and D2, no epistatic effects were detected between associated QTLs for grain width, panicle length, ratio of length to width and chalkiness degree (Fig. 4b, d, f, g) together with the other QTLs for plant height, number of primary branches, number of secondary branches, spikelet number per panicle, grain number per panicle, seed setting rate, yield per plant, head rice rate, chalky rice rate and gel consistency (data was not shown) .

\section{Substitution mapping and candidate gene analysis of $q G L 11$ and $q G W 5$}

The qGL 11 was firstly dissected into single segment substitution line (S6), whose estimated length of substitution segment was $1.42 \mathrm{Mb}$ and the maximum substitution length was $2.84 \mathrm{Mb}$ (from RM7173 to RM6085), Then 11 SSR markers were newly designed at the interval and 4 displayed polymorphism 
between Xihui 18 and Huhan 3. The electrophoresis bands for RM26001 and RM26038 were identical with those of Xihui 18, and RM26045 and RM26114 were same with Huhan 3. Thus, the estimated length and maximum length of the substitution segment of $\mathrm{S} 6$ were further shortened from the original $1.42 \mathrm{Mb}$ and 2.84 Mb to $1.415 \mathrm{Mb}$ and $1.66 \mathrm{Mb}$, respectively (from RM26038 to RM6085) (Fig. 5a). In the maximum substitution region of $q G L 11$, there are 2 genes related to phytohormones and ubiquitinproteasome pathway, namely LOC_Os11g04190 (OsPIN1C, auxin efflux carrier component) and LOC_Os11g04880 (ubiquitin-like protein 5). Three genes associated with cell length and number, including LOC_Os11g04350 (cell death associated protein), LOC_Os11g04580 (long cell-linked locus protein) and LOC_Os11g05850 (CycT1,3, rice cyclin gene). And 3 genes related to GRAS family transcription factor containing protein, including LOC_Os11g04400, LOC_Os11g04570 and LOC_Os11g04590. The other genes were not taken into account temporarily, including hypothetical proteins, unknown functional proteins, non-protein coding transcript and other family genes whose function was irrelevant to grain size. Therefore, we preliminarily selected these 8 genes as candidate genes of $q G L 11$. By DNA sequencing of these genes between Xihui 18 and S6, LOC_Os11g04350, LOC_Os11g04400, LOC_Os11g04580 and LOC_Os11g04880 showed no differences in promoter and exon regions between them, suggesting that these 4 genes were not candidate genes for $q G L 11$. Four genes displayed differences in DNA sequence between Xihui 18 and S6, including LOC_Os11g04190 (an auxin efflux carrier component), LOC_Os11g04570 and LOC_Os11g04590 (GRAS family transcription factor), LOC_Os11g05850 (CycT1;3, a rice cyclin gene) (Fig.5b-e). For LOC_Os11g04190, 5 single nucleotide polymorphism (SNP) differences in the coding DNA sequence (CDS) and 1 SNP difference in the 3' untranslated region (UTR) existed between Xihui 18 and S6. The $583^{\text {rd }}$ base of the CDS was changed from A of Xihui 18 to $\mathrm{G}$ of S6, which resulted in a mutation from Thr of Xihui 18 to Ala of S6. The other 4 SNP differences in CDS did not cause amino acid changes (Fig. 5b). For LOC_Os11g04570, 14 SNP differences in the CDS were detected, of which 9 caused amino acid mutations and 5 nonsense mutations (Fig. 5c). For LOC_Os11g04590, 8 SNP differences in the CDS were exhibited, of which 2 caused amino acid mutations and 6 nonsense mutations. In addition, (CCG) 7 repeat after the $180^{\text {th }}$ base of the CDS was in Xihui 18, while only (GGC) 6 repeat in S6, and the CCG encodes Proline (Fig. 5d). However, qRT-PCR analysis showed that the expression levels of the LOC_Os11g04190, LOC_Os11g04570 and LOC_Os11g04590 displayed no significant differences in root, stem, leaf, sheath and panicle between Xhui 18 and S6 (Fig. 5f-h). For LOC_Os11g05850 (CycT1;3), there were 6 SNP differences and a 25-base insertion in the 5'UTR and 1 SNP difference in the 3'UTR, and 1 SNP difference in the CDS which did not cause amino acid change and still encoded alanine (Fig. 5e). Especially, the expression level of the LOC_Os11g05850 was significantly higher in sheath and panicle in S6 than in Xihui18 (Fig. 5i). Thus, LOC_Os11g05850 (CyCT1;3) should be the best candidate gene for $q G L 11$, and LOC_Os11g04190, LOC_Os11g04570, LOC_Os11g04590 and LOC_Os11g05850 (CycT1;3) were potential candidate genes.

Using substitution mapping of S3, S4 and S5, qGW5 was delimited into $1.135 \mathrm{Mb}$ of the estimated substitution length and $1.33 \mathrm{Mb}$ of the maximum substitution interval between RM405 and RM17984 (Fig. 6a). At the interval, we found 6 genes involved in the reported signaling pathway related to grain 
size, such as phytohormones, ubiquitin-proteasome pathway and transcription factor regulation, including LOC_Os05g06270 (APIP6, RING E3 Ubiquitin Ligase), LOC_Os05g06280 (SRS3, small and round seed 3), LOC_Os05g06660 (GS5, regulator of grain size), LOC_Os05g06320 (OsERS2, ethylene receptor), LOC_Os05g06670 (OsGA2ox1, gibberellin 2-oxidase gene) and LOC_Os05g07720 (OsTAR1, IAA biosynthesis gene). The other genes as hypothetical proteins, unknown functional proteins and nonprotein coding transcripts were not temporarily taken into account. By DNA sequencing of these genes, only LOC_Os05g06660 (GS5) and LOC_Os05g07720 (OsTAR1) were found to be existed differences between Xihui 18 and S5. For LOC_Os05g06660(GS5), which was a regulator of grain size, (GGC) ${ }_{7}$ repeat after the $90^{\text {th }}$ base of the CDS were exsited in S5, while only (GGC) $)_{5}$ repeat in Xihui 18 , and the GGC encodes Glycine (Fig. 6b). For LOC_Os05g07720 (OsTAR1), which is an IAA biosynthesis gene, 4 SNP differences in the CDS were detected between Xihui18 and S5, of which 3 caused amino acid mutations and 1 nonsense mutations (Fig. 6c). Moreover, qRT-PCR analysis showed that the expression level of the LOC_Os05g06660 (GS5) was significantly higher in stem, leaf, sheath and panicle in S5 than in Xihui18, while no significant expression differences of the LOC_Os05g07720 (OSTAR1) were found in all organs between Xihui 18 and S5 (Fig. 6d, e). Thus, LOC_Os05g06660 (GS5) should be the best candidate gene for qGW5 and LOC_Os05g07720 (OsTAR1) were potential one for qGW5.

\section{Discussion}

\section{CSSL-Z414 and secondary SSSLs are ideal rice genetic study materials, and some are potential to be as restorer lines for hybrid rice breeding}

Restorer line plays an important role in heterosis utilization (Wu et al. 2018), and restorer lines are very important for breeding hybrid rice varieties. Xihui 18 was an indica restorer line bred by Southwest University. It had the characteristics of good combining ability, large panicle and multiple grains per panicle and narrow-long grain. In this study, we identified a short-wide grain CSSL-Z414 derived from Xihui 18 as the recipient parent. Z414 carried 4 chromosome substitution segments from donor Huhan 3. Again, none of their substitution segments contained the fertility restoration genes $R f-1$ (Akagii et al. 2004), Rf2 (Itabashi et al. 2011), Rf3 (Cai et al. 2013) and $R f 4$ (Cai et al. 2013), so Z414 are restorative. Z414 carried 9 QTLs for important traits and displayed short-wide and larger grain, shorter panicle and less number of primary branches, and higher brown rice rate and chalkiness degree. Thus, Z414 was not suited to be directly used as rice restorer line. While it is an ideal genetic study material for complex traits due to its consistent genetic background with Xihui18 except for only 4 substitution segments from Huhan 3. By genetic dissection, we obtained 6 single segment substitution lines (S1-S6) and 2 double segment substitution lines (D1-D2) harboring target QTLs. Intriguingly, S1 carried $q G L 3$ and $q C D 3$ and exhibited both long grain and lower chalkiness degree. D2 harbored both $q G L 3, q G L 11$ and $q C D 3$ and displayed shorter grain and lower chalkiness degree. Lower chalkiness degree is one of very important factors affected rice grain quality (Yang et al. 2021) and different grain size are import to meet the requirement of various people's preferences (Feng et al. 2021; Liang et al. 2021). Thus, some secondary substitution lines with significantly lower chalkiness degree than Xihui 18, such as S1 and D1 
are potential to be directly used as novel rice restorer lines to match with sterile lines. As for S5 with shortwide grain ( $q G L 5, q G W 5, q G W T 5, q R L W 5)$ and high chalkiness degree ( $q C D 5)$ and S6 with short grain ( $q G L 11, q R L W 11$ ), they can be used in researching the formation mechanism of these traits. Consequently, these SSSLS and DSSLS are important in both breeding and theoretical study.

\section{QTL identification in Z414 and SSSLs and genetic model analysis together with comparison to the reported genes}

Owing to Z414 still containing 8 difference traits from Xihui 18 and 4 substitution segments from donor parent Huhan 3. It is necessary for us to know which substitution loci control these traits and further dissect these loci into single segment substitution line. Finally, using the secondary $F_{2}$ population from Xihui 18/Z414 and 6 SSSLs (S1-S6), a total of 13 QTLs were found to be responsible for these difference traits, such as grain length, grain width, ratio of length to width, 1000-grain weight, panicle length, brown rice rate and chalkiness degree. Based on the analysis of these QTLs, Short grain of Z414 was controlled by $q G L 11, q G L 3$ and $q G L 5$, which was dissected into $S 6, S 1$ and $S 5$, respectively. Wide grain of Z414 was responsed by $q G W 5$, which was then validated in S4 and S5. Large grain of Z414 was in charge of $q G W T 5$ (verified by S4 and S5) and qGWT11 (verified by S6). Short panicle of Z414 was harbored by $q P L 3$ (validated by S1) and $q P L 11$. Higher brown rice rate was explained by $q B R R 1$. $q R L W 5$ and qRLW11 (verified by S4, S5 and S6) were responsible for the short-wide grain shape of Z414. qCD5 and $q C D 3$ (detected by S5 and S1) answered for the higher chalkiness degree. Thus, these special genetic materials could help us to understand the genetic models for different traits at specific genetic background and then to be applied in design breeding plan. As for each single homozygous segment substitution line, such as S6 carrying only qGL 11 for grain length, the genetic model of grain length at a specific environment is very simple, displaying only additive effects for the single homozygous locus at the backgrounds of Xihui 18. When for double homozygous segment substitution line, such as D2, the genetic model of grain length is the algebra sum of the additive effects $(A)$ of the single homozygous loci $q G L 11$ and $q G L 3$ and the A囚A epistatic interaction effect of the non-allelic homozygous loci ( $q G L 3$ and $q G L 11)$. For single heterozygous segment substitution line, the genetic model of heading date exists only dominant effect (D). For double heterozygous segment substitution line, the genetic model of heading date is the algebra sum of dominant effect (D) for each single heterozygous loci and the DषD epistatic interaction effect of the non-allelic heterozygous loci (Yang et al. 2018a). Thus, in natural $F_{1}$ hybrid materials, the genetic model is very complex exhibiting the combination of the above models. Genetic studies have shown that overdominance, incomplete dominance, and epistasis may all contribute to heterosis (Zhang et al. 2021b). Consequently, the development of single segment substitution lines and double segment substitution lines for different QTLs are very important to predict pyramid result of target QTL for precision design breeding. Studies have shown that pyramid different QTLs yielded different epistatic interaction. In this study, pyramid of $q G L 3(\mathrm{a}=0.22)$ and $q G L 11(\mathrm{a}=-0.19)$ yielded shorter grains $(\mathrm{G}=-0.28)$ in $\mathrm{D} 2$ than $\mathrm{S} 6$ (containing $q G L 11$ ), displaying $q G L 11$ was epistatic to $q G L 3$. Zhang et al (2020) showed that pyramid of $q G L 5(\mathrm{a}=0.30)$ and $q G L 6(\mathrm{a}=0.13)$ produced longer grains $(\mathrm{G}=0.74)$ of $\mathrm{D} 2$ than S2 and S3 at the genetic backgrounds of Nipponbare. Liang et al (2021) argued that pyramiding of 
different QTLs produces different epistatic interaction and their performance after pyramiding depends on the comparison between the algebraic sum of additive and epistatic effects of QTLs in the pyramidal line and the additive effect of all single QTLs. On the basis of the rule, so long as knowing the additive effects and epistatic effects of target QTLs at the specific environment, the phenotype of novel pyramided genotypes at specific environment can be predicted and then suitable genotypes can be selected according to our breeding goal, thus realizing the concept of design breeding.

Compared with the reported genes, OsAPC6, PLS2 and OspPLAllla can be as the candidate genes for qPL3 according to its physical distance and biological function. OSAPC6 interferes with the gibberellin signal pathway and leads to cell reduction (Awasthi et al. 2012). PLS2 encodes a glycosyltransferase, which participates in rice leaf senescence, and at the same time leads to shortening of internode and ear length (Wang et al. 2018). OspPLAIlla encodes glycoprotein-related phospholipase A and reduces panicle length (Liu et al. 2015b). According to the results in the study, qGW5 for grain width, $q G L 5$ for grain length, qRLW5 for ratio of length to width and qGWT5 for 1000-grain weight were within the interval of RM405 to RM17984 of chromosome 5, these QTLs should belong to pleiotropy. At the substitution interval, GS5 and OSTAR1 were potential candidate genes of these QTLs. Enhanced expression of GS5 inhibits the interaction between OSBAK1-7 and OSMSBP1, providing an explanation for the positive association between grain size and GS5 expression (Xu et al. 2015a). By DNA sequencing of GS5, we found that two GGC repeat encoding Glycine were added in CDS sequence of S5 compared with that of Xihui18. Again, qRT-PCR analysis showed that the expression level of the GS5 was significantly higher in stem, leaf, sheath and panicle in S5 than in Xihui18. OsTAR1 control the production of IAA in the developing rice grain, the ostar1 mutant has smaller grain (Xu et al. 2021). By DNA sequencing, there was 4 SNP differences in the CDS between Xihui18 and S5. While the expression of OsTAR1 displayed no significant differences between Xihui 18 and S5. Thus, GS5 should be the best candidate gene of qGW5. Whether GS5 and OSTAR1 were target genes of qGW5, the genetic complementary experiments of two genes were ongoing. Although GS5 and OSTAR1 have been cloned, we have found the alleles from Huhan 3 and developed the $S 5$ based on the genetic backgrounds of Xihui 18, which can be used in our plan of molecular design breeding. qGL3 was in the similar region with PGL1, OsLG3, TUD1 and OsOFP19. PGL 1 encodes an atypical basic helix-loop-helix protein (bHLH) that does not bind DNA. Its overexpression can increase grain length (Heang et al. 2012). OsLG3 positively regulates rice grain length and has no effect on grain quality (Yu et al. 2017). TUD1 encodes an E3 ubiquitin ligase of the U-box family, which participates in the brassinolide response and interacts with the heterotrimeric $\mathrm{G}$ protein subunit $D 1$ to regulate brassinolide-mediated rice growth (Hu et al. 2013). OSOFP19, OSH1 and DLT might form a functional complex that regulates cell proliferation and cell growth (Yang et al. 2018b). qCD3 was in the similar region with $G O T 1 B$, the mutations in the coding sequence of Golgi Transport 1 (GOT1B) cause high levels of endosperm $57 \mathrm{kDa}$ proglutelins and of the luminal chaperone binding protein (BiP) and increased grain chalkiness (Fukuda et al. 2016). qCD5 was in the similar region with Chalk5.

Chalk5 encodes a vacuolar H+-translocating pyrophosphatase (V-PPase), which increases the chalkiness of the endosperm by disturbing the $\mathrm{PH}$ homeostasis of the endomembrane trafficking system in 
developing seeds (Li et al. 2014). In addition, qPL11, qRLW11, qGWT11 and qBRR11 have not been reported.

\section{CycT1;3 should be the best candidate gene for $q G L 11$ whose function in regulating grain length was still unknown}

Elucidation of the molecular mechanism underlying grain size is important for rice design breeding. Considering genes associated with grain size that have been cloned, the majority involved in phytohormones pathway, G protein signal pathway, ubiquitin-proteasome pathway, Mitogen-activated protein kinase (MAPK) signal transduction pathway, and transcription factor regulation (Li et al. 2016). Within the maximum substitution interval of $1.66 \mathrm{Mb}$ for $q G L 11$ in $\mathrm{S} 6$, LOC_Os11g04190, LOC_Os11g04570, LOC_Os11g04590 and CycT1;3 which involved in the above signaling pathways might be candidate genes for qGL11. LOC_Os11g04190 is an unreported auxin efflux carrier component. IAA biosynthesis and transport is important for grain size. LOC_Os11g04570 and LOC_Os 11 g04590 are expressed proteins containing GRAS family transcription factors. GRAS family genes are usually related to plant hormones, and plant hormones play an important role in the molecular mechanism of grain size (Li et al, 2016). For example, SMOS2, a member of the GRAS family, can negatively regulate grain size by participating in the BR signaling pathway (Hirano et al. 2017). Moreover, By DNA sequencing, there were differences in both DNA sequence and Amino acids for LOC_Os11g04190, LOC_Os11g04570 and LOC_Os11g04590. While qRT-PCR analysis showed that the expression of three genes displayed no differences at any organs between Xihui 18 and S6. Thus, LOC_Os11g04190, LOC_Os11g04570 and LOC_Os11g04590 can only act as potential candidate genes for qGL 11. However, there was differences in both DNA sequence and gene expression in sheath and panicle for $\mathrm{CycT} 1 ; 3$ between Xihui 18 and S6. Thus, CyCT1,3 should be the best candidate gene for qGL 11. CyCT1;3 encodes cyclin protein, involving in biological progress of the cell Mitosis cycle. Intriguingly, cytological analysis showed that the shorter grain of Z414 depended on the decrease of cell number of glume rather than cell length. The result indicated that $q G L 11$ should be related to cell mitosis cycle. There were many studies on cell cycle regulation in yeast and animals, but few studies in plants. The biological process of the cell cycle is very complicated, cell proliferation in plants is mainly controlled by a family of cyclin-dependent kinases (CDKs). The activity of CDKs is directly regulated by binding and activation of cyclins (Wang et al. 2004; Gunbin et al. 2011). Plant cyclins can be regarded as a superfamily, which consists of several small families, such as A-, B-, C-, D-, H-, L-, T-, U-, SDS and J18 type Cyclin. The conserved regions of different types of cyclins in plants have different homology. There are more reports of $A, B, D$ and $E$ cyclins in plants (Nieduszynski et al. 2002), but still few reports on T cyclins, and there is a lack of systematic research on their expression patterns. Moreover, the orthologous homology of plant T-type cyclin and animal T-type protein is different, plant T-type cyclin forming a separate clade related to human T-type cyclin (Nieduszynski et al. 2002; La et al. 2006). Therefore, T-type cyclin in plant is worthy to further study. Qi et al (2012) found that GL3.1 encoding OsPPKL 1 can directly use Cyclin-T1;3 as a substrate to phosphorylate Cyclin-T1;3, which makes Cyclin-T1; 3 down-regulated in rice and resulting in shorter grains. But the molecular mechanism of how $C y c T 1 ; 3$ affects grain development remains unknown. In this study, although we detected $q G L 3$ for grain length in $S 1$, however, OSPPKL 1 was not in the institution 
interval of S1. On all accounts, these results are important for the in-depth study of $q G L 11$ and the series molecular design breeding based on backgrounds of Xihui 18 .

\section{Conclusions}

We identified a rice short-wide grain CSSL-Z414 carrying 4 substitution segments with average length of $3.04 \mathrm{Mb}$, which was derived from an indica restorer line Xihui18 as the recipient parent and Huhan 3 as the donor parent. Then, 9 QTLs were detected using the secondary $F_{2}$ population of Xihui $18 / Z 414$. And 6 QTLS ( $q P L 3, q G W 5, q G L 11, q R L W 5, q R L W 11, q G W T 5)$ could be validated by 6 SSSLs which were developed using MAS in $\mathrm{F}_{3}$ population. In addition, 4 QTLs ( $q G L 3, q G L 5, q C D 3$ and $\left.q C D 5\right)$ were detected by $S 1$ and $S 5$, which was not detected by the $F_{2}$ population. In particular, the chalkiness degree of $S 1$ was lower than that of Xihui 18, which is potential to be used as novel restorer lines. Pyramid of $q G L 3$ and $q G L 11$ yielded shorter grains in D2 than S6 (containing $q G L 11$ ), displaying $q G L 11$ was epistatic to qGL3. In D1 and D2, no epistatic effects were detected between related QTLs for grain width, panicle length, ratio of length to width and chalkiness degree. The grain length of Z414 was mainly controlled by $q G L 11, q G L 3$ and $q G L 5$. Cytological analysis, DNA sequencing and qRT-PCR analysis showed that CycT1,3 should be the best candidate gene for $q G L 11$, whose specific function regulating grain length was still unknown. The grain width of Z414 was controlled by $q G W 5$, and GS5 was the best candidate gene for $q G W 5$. These results laid a good foundation for the in-depth study for $C y c T 1,3$ regulating rice grain length and molecular design breeding based on backgrounds of Xihui 18.

\section{Materials And Methods}

\section{Materials}

Z414 was developed from Xihui 18 as the recipient parent and Huhan 3 as the donor parent. Xihui 18 was a rice excellent indica restorer line bred by Southwest University, with the characteristics of good combining ability, large panicle and multiple grains, and narrow-long grain. The japonica variety Huhan 3 had the characteristics of strong stress resistance and short-wide grain. Firstly, 429 simple sequence repeats (SSR) markers covering the whole rice genome were used to screen the polymorphisms between Xihui 18 and Huhan 3. Then, 241 polymorphic markers were selected to develop CSSLs beginning from $\mathrm{BC}_{2} \mathrm{~F}_{1}, 20$ plants in each generation were taken. Until in $\mathrm{BC}_{3} \mathrm{~F}_{7}$, a short-wide grain CSSL-Z414 with 4 substitution segments was identified in the study. The identification of chromosome substitution segments refers to the method described by Ma et al (2019), and the estimated length of chromosome substitution segments was calculated according to the method of Paterson et al (1991).

\section{Material planting}

Xihui 18 was crossed with Z414 at the experimental station of Southwest University, Chongqing, China in July of 2018, and the hybrid seeds were harvested and planted at the Hainan base in September of the same year and the $F_{1}$ seeds were harvested. Seeds of $Z 414$, Xihui 18 , and the $F_{2}$ population of 150 plants 
were sown on March 10, 2019 at the experimental station of Southwest University. Thirty seedlings of each parent and all $F_{1}$ seedlings were transplanted to the field on April 20, 2019, with 10 plants per row. The spacing between rows and individual plants were 26.4 and $16.5 \mathrm{~cm}$, respectively. Finally, the 12 individuals selected from $F_{2}$ population for developing SSSL and DSSL, as well as Z414 and Xihui 18, were planted at the experimental station of Southwest University on March 10, and transplanted on April 20, 2020, each material for 30 individuals. Conventional management practices were applied.

\section{Assessment of agronomic traits and quality parameters}

In the maturity period, 10 plants of Xihui 18 and Z414, together with 150 plants of $F_{2}$ were harvested. 13 traits were investigated including plant height, panicle number per plant, panicle length, number of primary branches, number of secondary branches, spikelets number per panicle, grains number per panicle, seed set rate, grain length, grain width, ratio of length to width, 1000-grain weight, and the yield per plant. The specific method referred to the described in Wang et al (2020).

The quality parameters referred to the national standard GB/T5495-2008. $10 \mathrm{~g}$ grains of Xihui 18 and $\mathrm{Z} 414$ and $150 \mathrm{~F}_{2}$ individuals were ground into brown rice. The brown rice was milled into polished rice using the CLS.JNM-1 rice husker. And then brown rice rate and head rice rate were calculated. The chalky rice rate and chalkiness degree were measured using all head rice for each sample by Wanshen SC-E. And the gel consistency was measured refer to the method described in Tang et al (1991).

The mean value of each trait was used in Student's $t$ test statistical analysis for comparison between Xihui 18 and Z414, and all parameters including mean value, standard deviation, skewness, kurtosis of these traits in the $F_{2}$ population was performed using statistical functions in Microsoft Excel 2016.

\section{Scanning electron microscope analysis of glumes in Z414 and Xihui 18}

At the completion of the booting stage and before the heading period, the inner and outer epidermal cells of the glume in Xihui18 and Z414 were investigated using a Hitachi SU3500 scanning electron microscope (Hitachi, Tokyo, Japan) with a frozen stage $\left(-40^{\circ} \mathrm{C}\right)$ under a low-vacuum environment.

\section{QTL mapping}

QTL mapping population was a secondary $F_{2}$ population consisted of 150 individuals derived from a cross between Xihui18 and Z414. The improved CTAB method described by McCouch et al (1988) was used to extract DNA from the parents and $150 \mathrm{~F}_{2}$ individuals. PCR amplification, polyacrylamide gel electrophoresis and rapid silver staining were carried out by the method described by Zhao et al (2016). Xihui 18 lanes were scored as " -1 ", Z414 lanes were scored as " 1 ", heterozygous lanes were scored as "0", and the absence of marker lanes was scored as ".". Lanes of each marker located on the substitution segment of $Z 414$, together with the phenotypic value of each individual of the $F_{2}$ population were used to identify putative QTL using the restricted maximum likelihood (REML) method implemented in the HPMIXED procedure in SAS (SAS Institute Inc., Cary, NC, USA). The P-value $<0.05$ was used as the 
threshold to determine whether a QTL could be linked to a certain marker on the substitution segment of Z414.

\section{Development of SSSLs and DSSLs, and validation and epistatic interaction analysis of target QTLs}

According to QTL mapping in 2019, 12 individuals carrying target QTL and no or less heterozygous markers selected from the $F_{2}$ were used to develop SSSL and DSSL by molecular marker-assisted selection (MAS) method. Each was planted as a line in 2020. Then, the leaves of 20 individuals for each line were taken to extract DNA to further develop SSSL and DSSL using the residual heterozygous markers in substitution segment by MAS. SSSL and DSSL obeyed the rule that each substitution line only carries the target substitution segment while the lanes of other markers were same with Xihui 18.

All individuals of SSSL and DSSL and 10 individuals of Xihui 18 were harvested after maturity in August of 2020. All the involved traits were measured again with the same methods in 2019. Since only one difference in single substitution segment was existed between each SSSL and Xihui18. Under certain environment (same year and same experimental field and no replicate plot designed), the genetic models for Xihui18 and SSSL carrying a specific QTL were: $P_{0}=\mu+\varepsilon$ and $P_{i}=\mu+a_{i}+\varepsilon$, respectively, where, $P_{0}$ and $P_{i}$ represent the phenotype value of any plant in plot of Xihui 18 and the $S S S L_{i}$ carrying the ' $i$ ' substitution segment. $\mu$ represent the mean value of Xihui18 population, $a_{i}$ represent additive effect of the $Q T L, \varepsilon$ represent random error. Thus, $t$-test was used to detect whether SSSL carry a QTL for a certain trait by comparing the statistical difference between each SSSL and Xihui 18, when the $P$-value was less than 0.05, a QTL was considered to be existed, and the additive effect of the QTL was calculated as half the difference between the mean phenotypic values of SSSL and Xihui18 (Wang et al. 2021). All calculations were conducted in Microsoft Excel 2016.

At the same environment, the genetic model for DSSL was: $P_{i j}=\mu+a_{i}+a_{j}+l_{i j}+\varepsilon$, where $P_{i j}$ represents the phenotype value of any plant in plot of the $D_{S S L_{i j}}, \mu$ represents the mean value of Xihui18 population, $a_{i}$ and $a_{j}$ represent the additive effect of QTL in substitution segment $i$ and $j$, respectively. $l_{i j}$ represent the $a_{i} a_{j}$ epistatic effect between QTLs in substitution segment $i$ and $j$. Thus, the epistatic effect between QTLs in DSSL was tested for the significance of each trait between (Xihui18+DSSL $\mathrm{ij}_{\mathrm{ij}}$ ) and $\left(\mathrm{SSSL}_{\mathrm{i}}+\mathrm{SSSL}_{\mathrm{j}}\right.$ ) using $t$ test, when the P-value is less than 0.05 , it is considered that there is an epistatic effect between the QTLs. The epistatic effects between non-allele QTLs were estimated as half of the mean phenotypic values of (Xihui18+DSSL $\mathrm{ij}_{\mathrm{ij}}$ )-(SSSL ${ }_{\mathrm{i}}+\mathrm{SSSL}_{\mathrm{j}}$ ) (Wang et al. 2021). Finally, we used IBM SPSS Statistics 25.0 to conduct multiple comparison.

\section{Candidate gene prediction and sequencing of $q G L 11$ and $q G W 5$}

Based on the result of QTL verification by SSSL, we synthesized new molecular markers to delimited the substitution interval. Candidate gene information within the maximum substitution of $q G L 11$ and $q G W 5$ was predicted and combined with gene annotations to select possible candidate genes with Gramene (http://www.gramene.org/rice_mutant/) and the China National Rice Database Center 
(http:/www.ricedata.cn/). The candidate gene sequence, including promoter, 5'UTR, exons, introns and 3 'UTR was downloaded, and the primers were designed on Vector NTI to amplify the target fragments using DNA of Xihui 18 and corresponding SSSL as templates, respectively. The amplicons were sequenced by Tsingke Biological Technology Co., Ltd. (Chongqing, China).

\section{Total RNA extraction and qRT-PCR analysis}

Total RNA was extracted from root, stem, leaf, sheath and panicle of Xihui 18 and SSSLs using the RNAprep Pure Plant RNA Purification Kit (Tiangen, Binjing, China) in the booting stage, and reverse transcribed using the GoScript Reverse Transcription System, and then analyzed quantitatively on a BioRad CYF96 using real-time PCR Master Mix (TaKaRa Biotechnology (Dalian, China) Co. Ltd.).

\section{Abbreviations}

QTL: Quantitative trait loci; CSSLs: Chromosome segment substitution lines; SSSL: Single-segment substitution lines; DSSL: Double-segment substitution lines; SSR: Simple sequence repeat; MAS: Markerassisted selection; PL: panicle length; GL: Grain length; GW: Grain width; RLW: Grain length-to-width ratio; GWT: 1000-grain weight; BRR: Brown rice rate ; CD: Chalkiness degree; SNP: Single nucleotide polymorphism; UTR: Untranslated region; CDS: Coding DNA sequence

\section{Declarations}

\section{Acknowledgments}

Professor Shizhong Xu (University of California, Riverside, USA) wrote the stem program for QTL mapping.

\section{Authors' Contribution}

FMZ designed the experiment and proposed the structure and content. JL, HXY, GYX, KLD, JJY, SQX, KZ, QLZ, RXL, MML and YHL performed experiment. FMZ, JL and GHH analyzed the data. ZLY was responsible for field management. JL and FMZ wrote the paper. All authors read and approved the final version.

\section{Funding}

The study was supported by National natural science foundation of China (31871593), the Chongqing technical innovation and application development Project (cstc2019jscx-msxmX0392).

\section{Availability of Data and Material}

The datasets supporting the conclusions of this article are included within the article.

\section{Ethics Approval and Consent to Participate}


This study complied with the ethical standards of China, where this research work was conducted.

\section{Consent for Publication}

All authors provide their consent for publication.

\section{Competing Interests}

The authors declare that they have no conflict of interest.

\section{References}

Akagi H, Nakamura A, Yokozeki-Misono Y, Inagaki A, Takahashi H, Mori K, Fujimura T (2004) Positional cloning of the rice $R f-1$ gene, a restorer of BT-type cytoplasmic male sterility that encodes a mitochondriatargeting PPR protein. Theor Appl Genet 108(8):1449-1457. doi:10.1007/s00122-004-1591-2

Awasthi A, Paul P, Kumar S, Verma SK, Prasad R, Dhaliwal HS (2012) Abnormal endosperm development causes female sterility in rice insertional mutant OsAPC6. Plant Sci 183:167-174. doi:10.1016/j.plantsci.2011.08.007

Cai J, Liao QP, Dai ZJ, Zhu HT, Zeng RZ, Zhang ZM, Zhang GQ (2013) Allelic differentiations and effects of the Rf3 and Rf4 genes on fertility restoration in rice with wild abortive cytoplasmic male sterility. Biol Plant 57(2):274-28. doi:10.1007/s10535-012-0294-9

Duan PG, Ni S, Wang JM, Zhang BL, Xu R, Wang YX, Chen HQ, Zhu XD, Li YH (2015) Regulation of OsGRF4 by OsmiR396 controls grain size and yield in rice. Nat Plants 2(1):15203. doi:10.1111/pbi.12569

Feng Y, Yuan XP, Wang YP, Yang YL, Zhang MC, Yu HY, Xu Q, Wang S, Niu XJ, Wei XH (2021) Validation of a QTL for grain size and weight using an introgression line from a cross between Oryza sativa and Oryza minuta. Rice, 14(1): 43. Doi:10.1186/s12284-021-00472-1

Fukuda M, Kawagoe Y, Murakami T, Washida H, Sugino A, Nagamine A, Okita TW, Ogawa W, Kumama T (2016) The Dual Roles of the Golgi Transport 1 (GOT1B): RNA localization to the cortical endoplasmic reticulum and the export of proglutelin and a-Globulin from the cortical ER to the colgi. Plant Cell Physiol 57(11): 2380-2391

Gao XY, Zhang JQ, Zhang XJ, Zhou J, Jiang ZH, Huang P, Tang ZB, Bao YM, Cheng JP, Tang HJ, Zhang WH, Zhang HS, Huang J (2019) Rice qGL3/OSPPKL 1 functions with the GSK3/SHAGGY-Like Kinase OsGSK3 to modulate brassinosteroid signaling. Plant Cell 31(5):1077-1093. doi:10.1105/tpc.18.00836

Gunbin KV, Suslov VV, Turnaev II, Afonnikov DA, Kolchanov NA (2011) Molecular evolution of cyclin proteins in animals and fungi, BMC Evol Biol 11(1):224. doi:10.1186/1471-2148-11-224 
Guo T, Chen K, Dong NQ, Shi CL, Ye WW, Gao JP, Shan JX, Lin HX (2018) Grain size and number negatively regulates the OSMKKK10-OSMKK4-OSMPK6 cascade to coordinate the trade-off between grain number per panicle and grain size in rice. Plant Cell 30(4):871-888. doi:10.1105/tpc.17.00959

Heang D, Sassa $\mathrm{H}$ (2012) Antagonistic actions of $\mathrm{HLH} / \mathrm{bHLH}$ proteins are involved in grain length and weight in rice. PLoS ONE 7(2): e31325. doi:10.1371/journal.pone.0031325

Hirano K, Yoshida H, Aya K, Kawamura M, Hayashi M, Hobo T, Sato-lzawa K, Kitano H, Ueguchi-Tanaka M, Matsuoka M (2017) SMALL ORGAN SIZE 1 and SMALL ORGAN SIZE 2/ DWARF AND LOW-TILLERING form a complex to integrate auxin and brassinosteroid signaling in rice. Mol Plant 10(4): 590-604. doi:10.1016/j.molp.2016.12.013

Hu XM, Qian Q, Xu T, Zhang Y, Dong GJ, Gao T, Xie Q, Xue YB (2013) The U-Box E3 ubiquitin ligase TUD1 functions with a heterotrimeric Ga subunit to regulate brassinosteroid-mediated growth in rice. PLoS Genet 9(3): e1003391. doi:10.1371/journal.pgen.1003391

Huang RY, Jiang LG, Zheng JS, Wang TS, Wang HC, Huang YM, Hong ZL (2013) Genetic bases of rice grain shape: so many genes, so little known. Trends Plant Sci 18(4):218-226.

doi:10.1016/j.tplants.2012.11.001

Ishimaru K, Hirotsu N, Madoka Y, Murakami N, Hara N, Onodera H, Kashiwagi T, Ujiie K, Shimizu B, Onishi A, Miyagawa $H$, Katoh E (2013) Loss of function of the IAA-glucose hydrolase gene TGW6 enhances rice grain weight and increases yield. Nat Genet 45(6):707-711. doi:10.1038/ng.2612

Itabashi E, Iwata N, Fujii S, Kazama T, Toriyama K (2011) The fertility restorer gene, $R f 2$, for lead rice-type cytoplasmic male sterility of rice encodes a mitochondrial glycine-rich protein. Plant J 65(3):359-367. doi:10.1111/j.1365-313X.2010.04427.x

La HG, Li J, Ji ZD, Cheng YJ, Li XL, Jiang SY, Venkatesh PN, Ramachandran S (2006) Genome-wide analysis of cyclin family in rice (Oryza Sativa L.). Mol Genet Genomics 275(4):374-386. doi:10.1007/s00438-005-0093-5

Li N, Li YH (2016) Signaling pathways of seed size control in plants. Curr Opin Plant Biol 33: 23-32. doi:10.1016/j.pbi.2016.05.008

Li YB, Fan CC, Xing YZ, Jiang YH, Luo LJ, Sun L, Shao D, Xu CJ, Li XH, Xiao JH, HeYQ, Zhang QF (2011) Natural variation in GS5 plays an important role in regulating grain size and yield in rice. Nature Genetics, 2011, 43(12): 1266-1269 doi: 10.1038/ng.977

Li YB, Fan CC, Xing YZ, Yun P, Luo LJ, Yan B, Peng B, Xie WB, Wang GW, Li XH, Xiao JH, Xu CG, He YQ (2014) Chalk5 encodes a vacuolar $\mathrm{H}+$-translocating pyrophosphatase influencing grain chalkiness in rice. Nat Genet 46(4): 398-404. 
Liang PX, Wang H, Zhang QL, Zhou K, Li MM, Li RX, Xiang SQ, Zhang T, Ling YH, Yang ZL, He GH, Zhao FM (2021) Identification and pyramiding of QTLs for rice grain size based on short-wide grain CSSL-Z563 and fine-mapping of $q G L 3-2$. Rice, 14(1): 35. doi:10.1186/s12284-021-00477-w

Liu GM, Zhang K, Ai J, Deng XJ, Hong YY, Wang XM (2015b) Patatin-related phospholipase A, pPLAIIla, modulates the longitudinal growth ofvegetative tissues and seeds in rice. J Exp Bot 66(21):6945-6955. doi:10.1093/jxb/erv402

Liu JF, Chen J, Zheng XM, Wu FQ, Lin QB, Heng YQ, Tian P, Cheng ZJ, Yu XW, Zhou KN, Zhang X, Guo XP, Wang JL, Wang HY, Wan JM (2017) GW5 acts in the brassinosteroid signalling pathway to regulate grain width and weight in rice. Nat Plants 3(5): 17043. doi:10.1038/nplants.2017.43

Liu L, Tong HN, Xiao YH, Che RH, Xu F, Hu B, Liang CZ, Chu J F,Li JY, Chu CC (2015a) Activation of Big Grain 1 significantly improves grain size by regulating auxin transport in rice. Proc Natl Acad Sci USA 112(35):11102-11107. doi:10.1073/pnas.1517098112

Ma F Y, Zhu XY, Wang H, Wang SM, Cui GQ, Zhang T, Yang ZL, He GH, Ling YH, Wang N, Zhao FM (2019) Identification of QTL for kernel number-related traits in a rice chromosome segment substitution line and fine mapping of qSP1. Crop J 7(4):494-503. doi:10.1016/j.cj.2018.12.009

McCouch SR, Kochert G, Yu ZH, Wang ZY, Khush GS, Coffman WR, Tanksley SD (1988) Molecular mapping of rice chromosome. Theor Appl Genet 76(6):815-829. doi:10.1007/BF00273666

Nieduszynski CA, Murray J, Carrington M (2002) Whole-genome analysis of animal A- and B-type cyclins. Genome Biol 3(12):1164-1176. doi:10.1186/gb-2002-3-12-research0070

Paterson AH, Damon S, Hewitt JD, Zamir D, Rabinowitch HD, Lincoln SE, Lander ES, Tanksley SD (1991) Mendelian factors underlying quantitative traits in tomato: comparison across species, generations, and environments. Genetics 127(1):181-197.

Qi P, Lin YS, Song XJ, Shen JB, Huang W, Shan JX, Zhu MZ, Jiang LW, Gao JP, Lin HX (2012) The novel quantitative trait locus GL3.1 controls rice grain size and yield by regulating Cyclin-T1;3. Cell Res 22(12):1666-1680. doi:10.1038/cr.2012.151

Shi CL, Ren YL, Liu LL, Wang F, Zhang H, Tian P, Pan T, Wang YF, Jing RN, Liu TZ, Wu FQ, Lin QB, Lei CL, Zhang X, Zhu SH, Guo XP, Wang JL, Zhao ZC, Wang J, Zhai HQ, Cheng ZJ, Wan JM (2019) Ubiquitin specific protease 15 has an important role in regulating grain width and size in rice. Plant Physiol 180(1): 381-391. doi:10.1104/pp.19.00065

Song XJ, Huang W, Shi M, Zhu MZ, Lin HX (2007) A QTL for rice grain width and weight encodes a previously unknown RING-type E3 ubiquitin ligase. Nat Genet 39(5): 623-63. doi.:10.1038/ng2014

Sun SY, Wang L, Mao HL, Shao L, Li XH, Xiao JH, Ouyang YD, Zhang QF (2018) A G-protein pathway determines grain size in rice. Nat Commun 9(1): 851. doi:10.1038/s41467-018-03141-y 
Tang SX, Khush GS, Juliano BO (1991) Genetics of gel consistency in rice (Oryza sativa L.). J Genet 70(2):69-78. doi:10.1007/BF02927807

Wang DC, Zhou K, Xiang SQ, Zhang QL, Li RX, Li MM, Liang PX, Farkhanda N, He GH, Ling YH, Zhao FM(2021) Identification, pyramid and candidate genes of QTLs for associated traits based on a dense erect panicle rice CSSL-Z749 and five SSSLs, three DSSLs and One TSSL. Rice 149(1): 55. doi:10.1186/s12284-021-00496-7

Wang GF, Kong HZ, Sun YJ, Zhang XH, Zhang W, Altman NM, dePamphilis CW, Ma H (2004) Genome-wide analysis of the cyclin family in arabidopsis and comparative phylogenetic analysis of plant cyclin-like proteins. Plant Physiol 135(2):1084-1099. doi:10.1104/pp.104.040436

Wang H, Zhang JY, Farkhanda N, Li J, Sun SF, He GH, Zhang T, Ling YH, Zhao FM (2020) Identification of rice QTLs for important agronomic traits with long-kernel CSSL-Z741 and three SSSLs. Rice Sci 27(5)414423. doi:10.1016/j.rsci.2020.04.008

Wang M, Zhang T, Peng H, Luo S, Tan JJ, Jiang KF, Heng YQ, Zhang X, Guo XP, Zheng JK, Cheng ZJ (2018) Rice premature leaf senescence 2, encoding a glycosyltransferase (GT), is involved in leaf senescence. Front Plant Sci 9: 560. doi:10.3389/fpls.2018.00560

Wang SK, Li S, Liu Q, Wu K, Zhang JQ, Wang SS, Wang Y, Chen XB, Zhang Y, Gao CX, Wang F, Huang HX, Fu XD (2015) The OSSPL 16-GW7 regulatory module determines grain shape and simultaneously improves rice yield and grain quality. Nat Genet 47(8):949-954. doi:10.1038/ng.3352

Wu B, Hu W, Xing YZ (2018) The history and prospect of rice genetic breeding in China. Hereditas 40(10):841-857. (in Chinese with English abstract). doi:10.16288/j.yczz.18-213

Xu CJ, Liu Y, Li YB, Xu XD, Xu CG, Li XH, Xiao JH, Zhang QF (2015a) Differential expression of GS5 regulates grain size in rice. J Exp Bot 66(9):2611-2623. doi:10.1093/jxb/erv058

Xu J, Zhang SQ (2015b) Mitogen-activated protein kinase cascades in signaling plant growth and development. Trends Plant Sci 20(1):56-64. doi:10.1016/j.tplants.2014.10.001

Xu R, Duan PG, Yu HY, Zhou ZK, Zhang BL, Wang RC, Li J, Zhang GZ, Huang SS, Lyu J, Li N, Chai TY, Tian ZX, Yao SG, Li YH (2018) Control of grain size and weight by the OsMKKK10-OsMKK4-OsMAPK6 signaling pathway in rice. Mol Plant 11(6):860-873. doi:10.1016/j.molp.2018.04.004

Xu XY, E ZG, Zhang DP, Yun QB, Zhou Y, Niu BX, Chen C (2021) OsYUC11-mediated auxin biosynthesis is essential for endosperm development of rice. Plant Physiol 185(3): 934-950. doi:10.1093/plphys/kiaa057

Yang C, Ma YM, He Y, Tian ZH, Li JX (2018b) OsOFP19 modulates plant architecture by integrating the cell division pattern and brassinosteroid signaling. Plant J 93(3):489-501. doi:10.1111/tpj.13793 
Yang WF, Liang JY, Hao QW, Luan X, Tan QY, Lin SW, Zhu HT, Liu GF, Liu ZP, Bu SH, Wang SK, Zhang GQ (2021) Fine mapping of two grain chalkiness QTLs sensitive to high temperature in rice. Rice 14(1):33. doi:10.1186/s12284-021-00476-x

Yang ZF, Jin LL, Zhu HT, Wang SK, Zhang GQ, Liu GF (2018a) Analysis of epistasis among QTLs on heading date based on single segment substitution lines in rice. Sci Rep 8(1): 3059. doi:10.1038/s41598018-20690-w

Yu J, Xiong HY, Zhu XY, Zhang HL, Li HH, Miao JL, Wang WS, Tang ZS, Zhang ZY, Yao GX, Zhang Q, Pan YH, Wang X, Rashid MAR, Li JJ, Gao YM, Li ZK, Yang WC, Fu XD, Li ZC (2017) OsLG3 contributing to rice grain length and yield was mined by Ho-LAMap. BMC Biol 15(1):28. doi:10.1186/s12915-017-0365-7

Zhang GQ(2021a) Target chromosome-segment substitution: A way to breeding by design in rice. Crop J 9(03): 658-668. doi:10.1016/j.cj.2021.03.001

Zhang SN, Huang XH, Han B (2021b) Understanding the genetic basis of rice heterosis: Advances and prospects, Crop Journal 9(03): 688-692. doi:10.1016/j.cj.2021.03.011

Zhang T, Wang SM, Sun SF, Zhang Y, Li J, You J, Su T, Chen WB, Ling YH, He GH, Zhao FM (2020) Analysis of QTL for grain size in a rice chromosome segment substitution line Z1392 with long grains and fine mapping of $q G L-6$. Rice 13(1): 30. Doi:10.1186/s12284-020-00399-z

Zhao FM, Tan Y, Zheng LY, Zhou K, He GH, Ling YH, Zhang LH, Xu SZ (2016) Identification of rice chromosome segment substitution line Z322-1-10 and mapping QTLs for agronomic traits from the $F_{3}$ population. Cereal Res Commun 44(3):370-380. doi:10.1556/0806.44.2016.022

\section{Tables}

Table 1 -Statistical parameters of different traits in Xihui18, Z414, and the $F_{2}$ population 


\begin{tabular}{|c|c|c|c|c|c|c|}
\hline \multirow[t]{2}{*}{ Trait } & \multicolumn{2}{|c|}{ Mean $\pm S D$ (parents) } & \multicolumn{4}{|l|}{$\mathrm{F}_{2}$ population } \\
\hline & Xihui 18 & Z414 & Mean $\pm S D$ & Range & Skew & Kurt \\
\hline Plant height $(\mathrm{cm})$ & $133.87 \pm 3.48$ & $130.90 \pm 4.24$ & $132.67 \pm 5.88$ & $\begin{array}{l}118.70- \\
147.80\end{array}$ & -0.07 & 0.14 \\
\hline Panicle number & $4.22 \pm 0.66$ & $4.13 \pm 0.93$ & $5.22 \pm 2.45$ & $\begin{array}{l}2.00- \\
25.00\end{array}$ & 4.59 & 32.83 \\
\hline Panicle length $(\mathrm{cm})$ & $27.03 . \pm 1.1$ & $25.03 \pm 0.73 * \star$ & $25.85 \pm 1.70$ & $\begin{array}{l}19.78- \\
31.06\end{array}$ & -0.05 & 1.19 \\
\hline $\begin{array}{l}\text { Number of primary } \\
\text { branches }\end{array}$ & $16.62 \pm 0.73$ & $13.67 \pm 0.56^{\star \star}$ & $14.69 \pm 1.22$ & $\begin{array}{l}10.00- \\
17.67\end{array}$ & -0.49 & 1.73 \\
\hline $\begin{array}{l}\text { Number of } \\
\text { secondary } \\
\text { branches }\end{array}$ & $42.28 \pm 6.14$ & $36.90 \pm 4.18$ & $36.66 \pm 8.66$ & $\begin{array}{l}18.60- \\
62.67\end{array}$ & 0.28 & 0.32 \\
\hline $\begin{array}{l}\text { Spikelet number } \\
\text { per panicle }\end{array}$ & $201.68 \pm 25.66$ & $177.16 \pm 22.84$ & $182.08 \pm 34.02$ & $\begin{array}{l}110.33- \\
307.89\end{array}$ & 0.32 & 0.81 \\
\hline $\begin{array}{l}\text { Grain number per } \\
\text { panicle }\end{array}$ & $229.41 \pm 27.33$ & $201.49 \pm 23.15$ & $203.72 \pm 37.06$ & $\begin{array}{l}124.60- \\
338.78\end{array}$ & 0.28 & 0.84 \\
\hline $\begin{array}{l}\text { Seed setting rate } \\
(\%)\end{array}$ & $88.21 \pm 0.20$ & $89.05 \pm 0.10$ & $89.23 \pm 3.62$ & $\begin{array}{l}69.03- \\
95.32\end{array}$ & -2.33 & 8.93 \\
\hline Grain length (mm) & $10.22 \pm 0.08$ & $9.43 \pm 0.11$ ** & $9.74 \pm 0.17$ & $\begin{array}{l}9.17- \\
10.27\end{array}$ & 0.07 & 0.47 \\
\hline Grain width (mm) & $3.02 \pm 0.02$ & $3.73 \pm 0.07 * \star$ & $3.37 \pm 0.17$ & $\begin{array}{l}2.93- \\
3.70\end{array}$ & -0.22 & -0.19 \\
\hline $\begin{array}{l}\text { Ratio of Length to } \\
\text { width }\end{array}$ & $3.38 \pm 0.03$ & $2.53 \pm 0.06$ ** & $2.90 \pm 0.16$ & $\begin{array}{l}2.56- \\
3.29\end{array}$ & 0.33 & -0.17 \\
\hline $\begin{array}{l}\text { 1000-grain weight } \\
\text { (g) }\end{array}$ & $28.96 \pm 0.86$ & $33.63 \pm 0.49 \star \star$ & $31.69 \pm 1.77$ & $\begin{array}{l}27.40- \\
36.37\end{array}$ & 0.29 & -0.31 \\
\hline Yield per plant (g) & $24.82 \pm 4.82$ & $21.82 \pm 4.84$ & $30.69 \pm 17.07$ & $\begin{array}{l}12.40- \\
147.37\end{array}$ & 3.56 & 18.42 \\
\hline Brown rice rate (\%) & $69.03 \pm 2.49$ & $75.11 \pm 4.31$ *夫 & $74.12 \pm 4.05$ & $\begin{array}{l}69.12- \\
83.05\end{array}$ & 3.80 & 28.95 \\
\hline Head rice rate $(\%)$ & $28.13 \pm 2.36$ & $25.21 \pm 1.78$ & $25.21 \pm 6.38$ & $\begin{array}{l}10.11-y \\
\text { the } \\
43.22\end{array}$ & 0.38 & 0.15 \\
\hline Chalky rice rate (\%) & $99.19 \pm 1.60$ & $100.00 \pm 0.00$ & $99.01 \pm 1.78$ & $\begin{array}{l}92.00- \\
100.00\end{array}$ & -1.92 & 3.30 \\
\hline $\begin{array}{l}\text { Chalkiness degree } \\
(\%)\end{array}$ & $19.98 \pm 1.76$ & $25.29 \pm 1.28 * \star$ & $24.18 \pm 2.04$ & $\begin{array}{l}18.84- \\
29.39\end{array}$ & -0.19 & -0.13 \\
\hline
\end{tabular}


* and ** indicate significant difference between traits of Xihui 18 and $Z 414$ at $P<0.05$ and $P<0.01$, respectively.

Table 2-QTL for agronomic and quality traits identified in substitution segments of Z414

\begin{tabular}{|lllllll|}
\hline Trait & QTL & Chr. & Linked marker & Additive effect & $\mathrm{R}^{2}(\%)$ & P-value \\
\hline Panicle length (cm) & qPL3 & 3 & RM3766 & -0.58 & 9.02 & 0.0295 \\
\hline Panicle length (cm) & qPL11 & 11 & RM1812 & 0.51 & 7.33 & 0.0063 \\
\hline Grain width $(\mathrm{mm})$ & qGW5 & 5 & RM5874 & 0.12 & 50.39 & 0.0013 \\
\hline Grain length (mm) & qGL11 & 11 & RM1812 & -0.08 & 9.96 & 0.0138 \\
\hline Ratio of length to width & qRLW5 & 5 & RM5874 & -0.10 & 38.38 & 0.0038 \\
\hline Ratio of length to width & qRLW11 & 11 & RM1812 & -0.04 & 7.15 & 0.0069 \\
\hline 1000-grain weight (g) & qGWT5 & 5 & RM5874 & 0.63 & 10.47 & 0.0450 \\
\hline 1000-grain weight $(\mathrm{g})$ & qGWT11 & 11 & RM1812 & -0.37 & 3.86 & 0.0457 \\
\hline Brown rice rate $(\%)$ & qBRR11 & 11 & RM1812 & 1.19 & 5.92 & 0.0309 \\
\hline
\end{tabular}

\section{Figures}


Chr.3

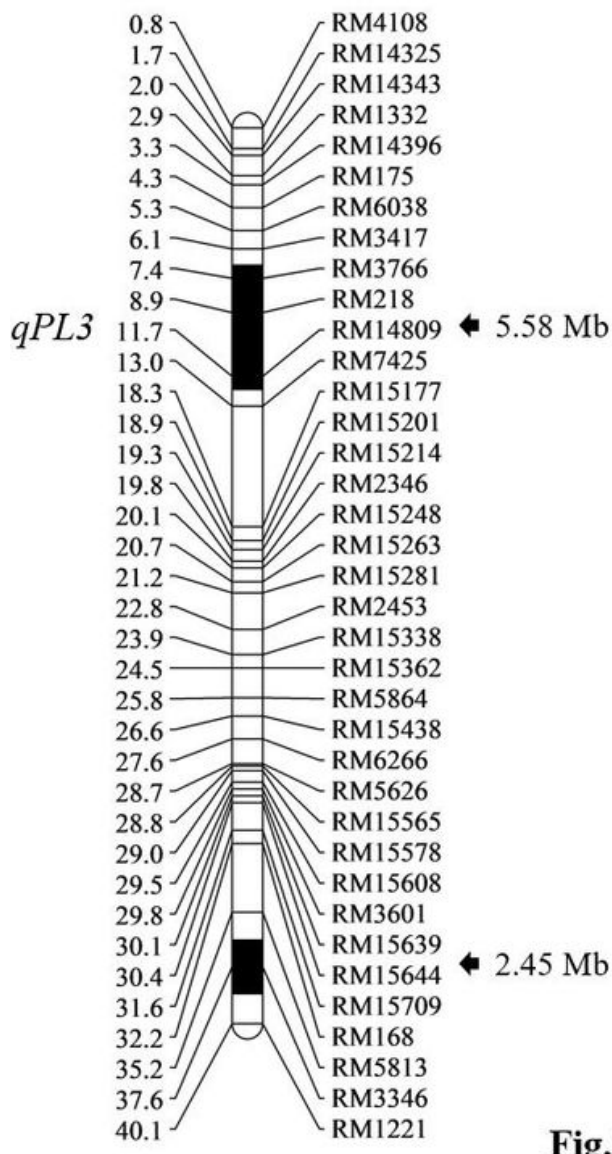

Chr.5

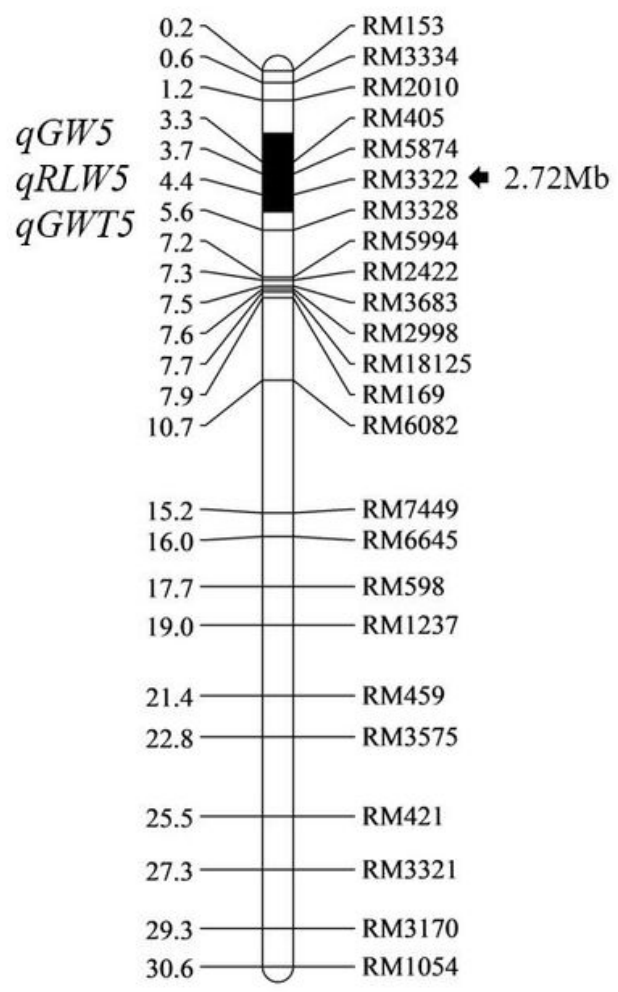

Fig.1. Chromosome substitution segments of Z414
Chr.11

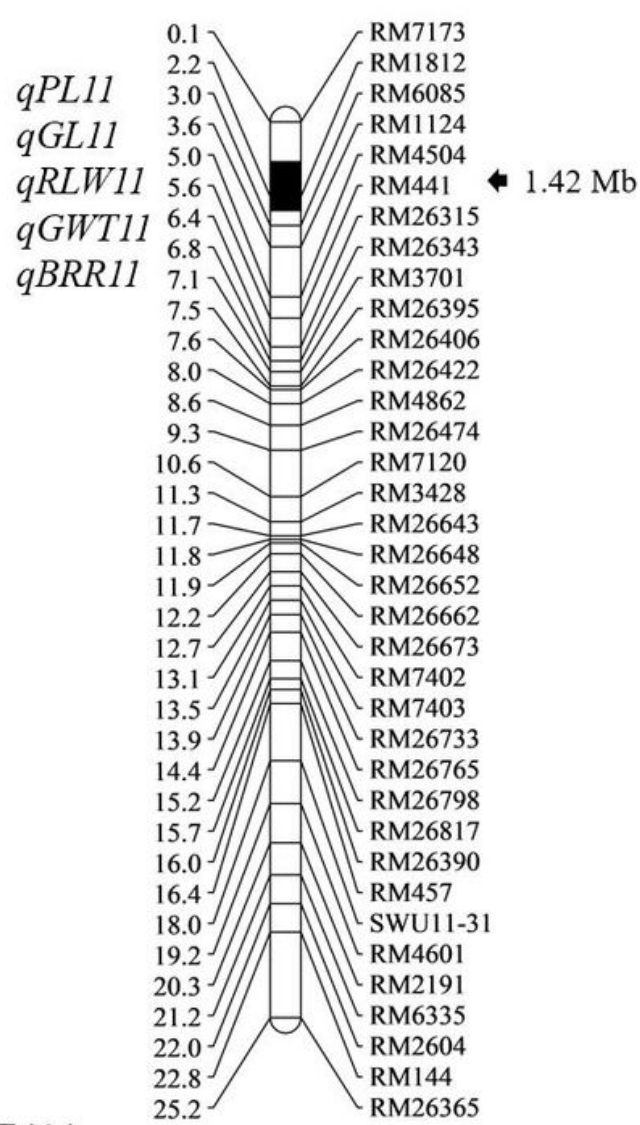

Figure 1

Chromosome substitution segments of Z414. Physical distances (Mb) and mapped QTL are marked at left , markers and substitution segment length are displayed at the right. Black section on each chromosome are substitution segments. PL, Panicle length; GW, Grain width; GL, Grain length; RLW, ratio of length to width; GWT, 1000-Grain weight; BRR, Brown rice rate. 


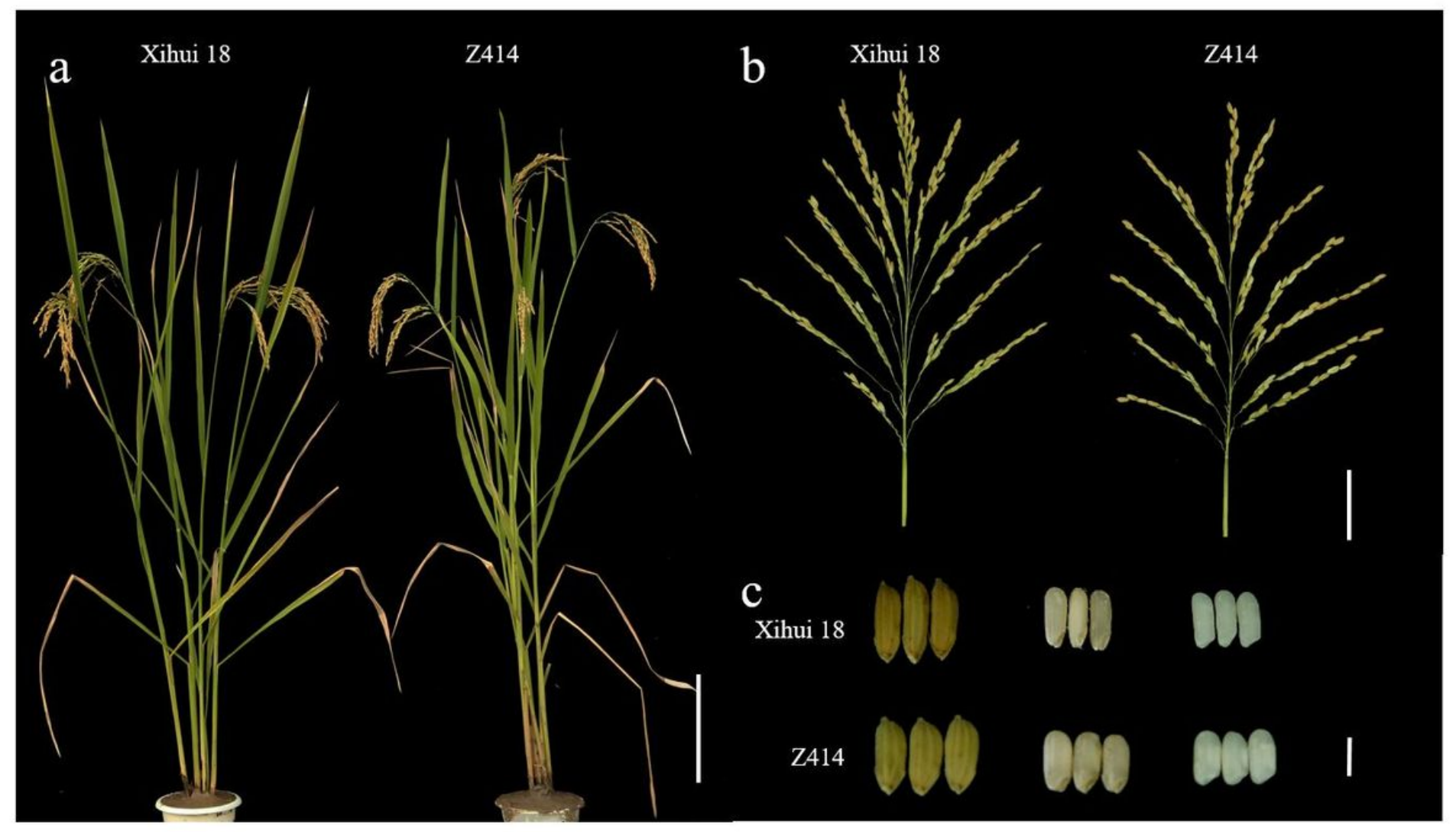

Figure 2

Phenotype of Xihui 18 and Z414. a Plant type of Xihui 18 and Z414. b Panicle of Xihui 18 and Z414. c Grain, brown rice and polished rice of Xihui 18 and Z414. Bars in a $20 \mathrm{~cm}$, b 5 cm; c 5mm. 

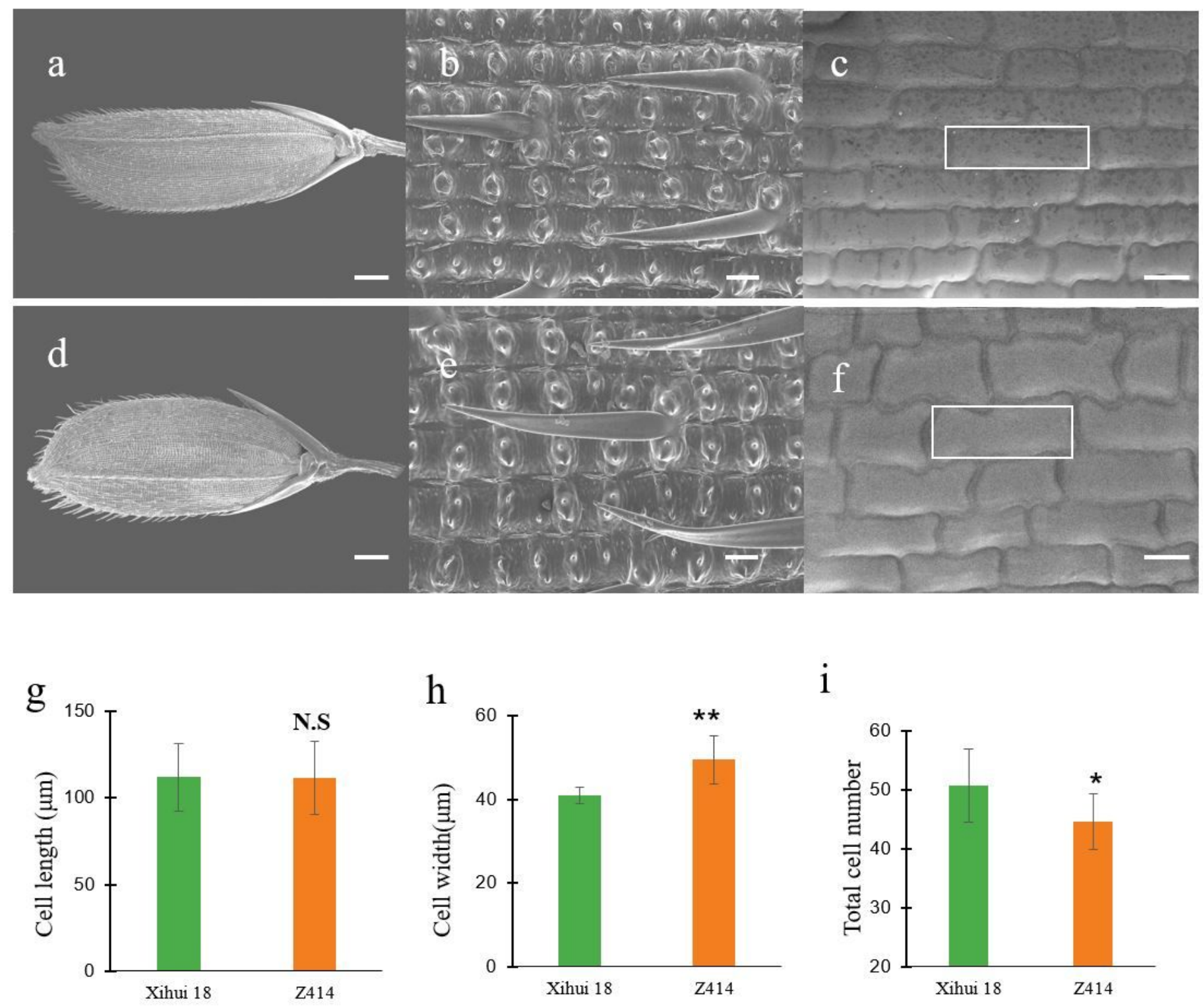

$\mathrm{i}$

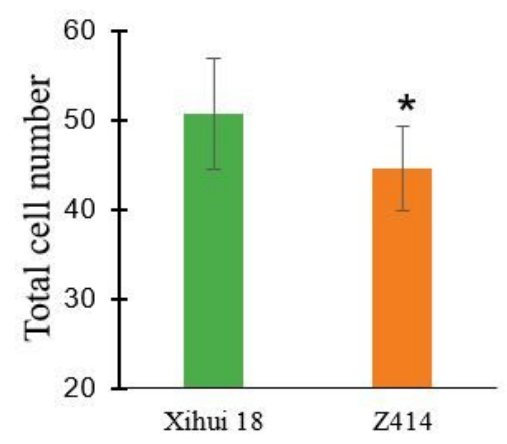

Figure 3

Scanning electron microscopy observation and analysis of glume in Xihui 18 and Z414. a-f Scanning electron microscope of the lemma ( $a, d)$, outer epidermis $(b, e)$, and inner epidermis $(c, f)$ of the glume of Xihui 18(a-c) and Z414(d-f). g, h and i represent cell length, width and total number of cells in outer epidermis of lemma at 200x magnification, respectively. ${ }^{* *}$ and *respectively indicates difference at 0.01 and 0.05 level between Xihui 18 and Z414. Bars in a and d $10 \mathrm{~mm}, \mathrm{~b}$ and e $500 \mu \mathrm{m}, \mathrm{c}$ and $\mathrm{f} 500 \mu \mathrm{m}$. 
$\mathbf{a}$

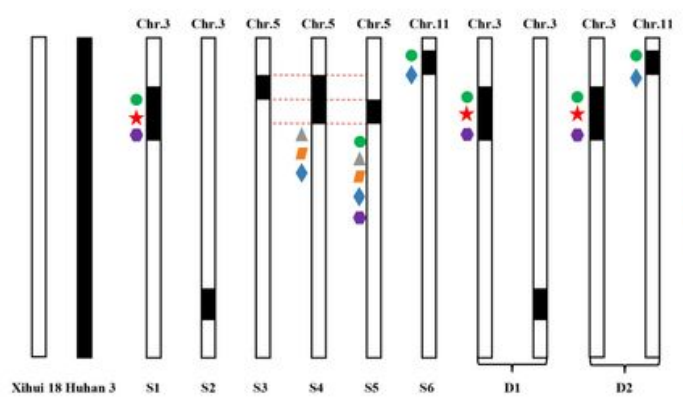

b

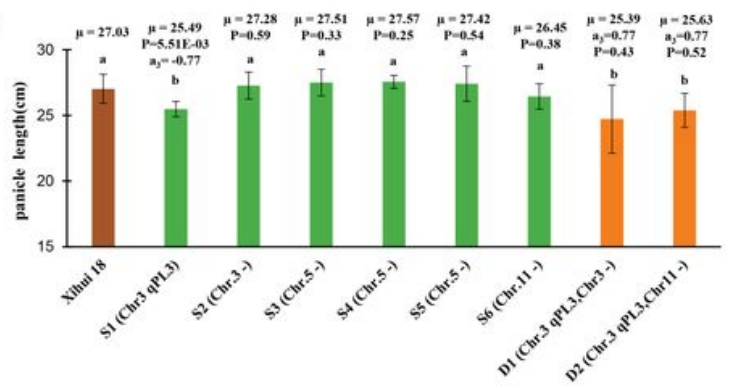

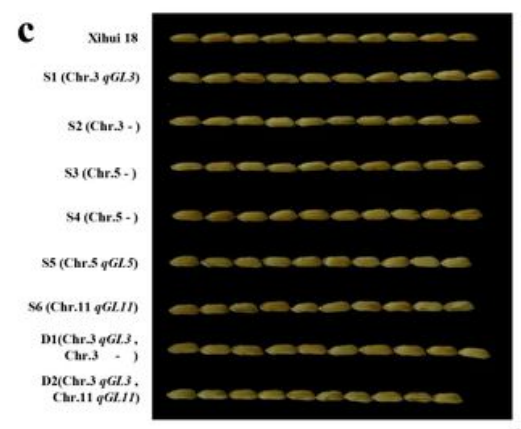

e

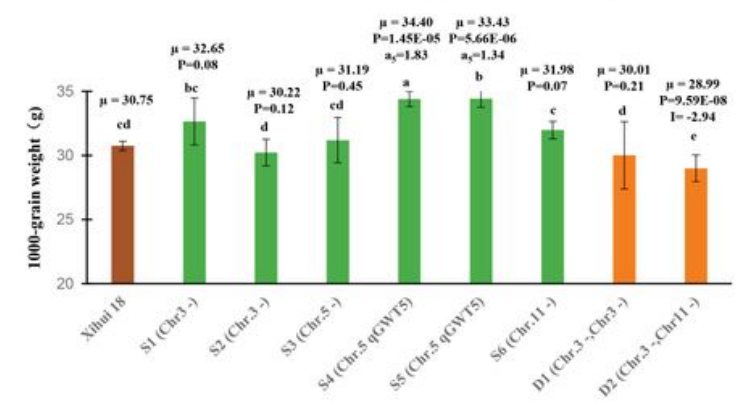

g
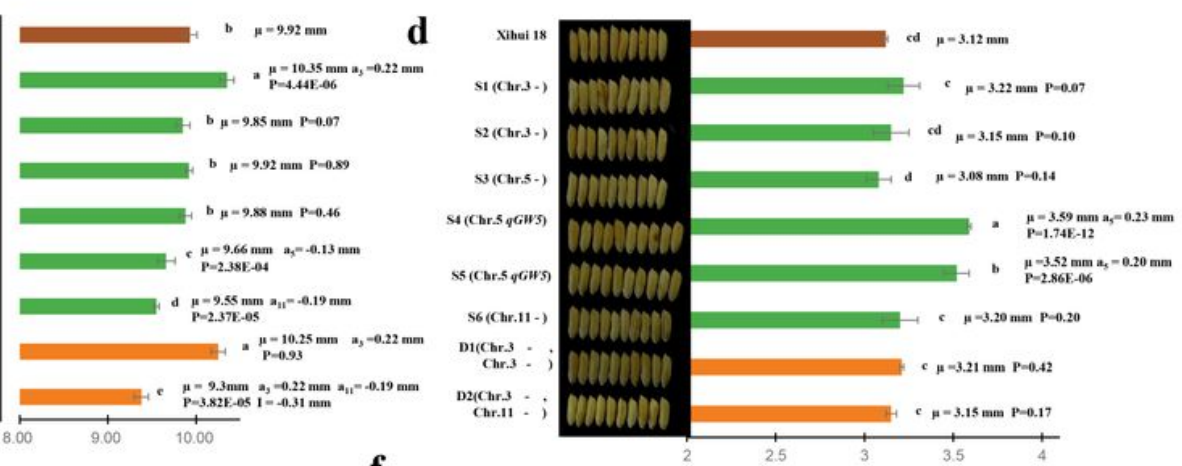

f

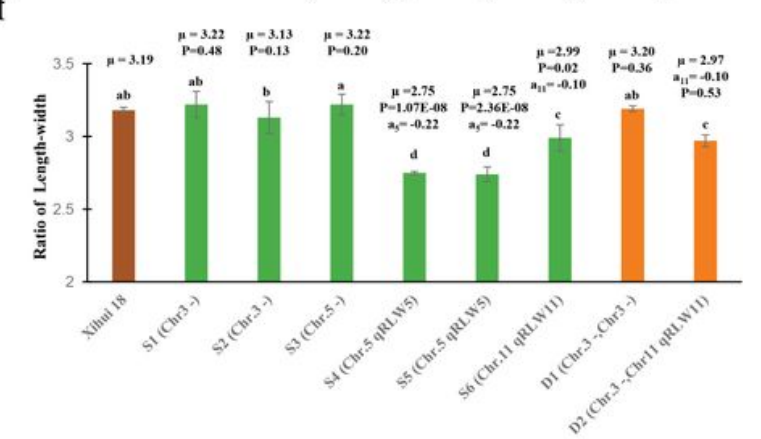

\section{Figure 4}

Additive and epistatic effect of QTLs for related traits in SSSL, DSSL and their phenotype. a Schematic diagram of substitution segment and QTL located on them of S1-S4, and D1 and D2; b-f Parameters of QTLs in different SSSLs and DSSLs for panicle length (b), Grain length(c), Grain width (d), 1000-grain weight (e), ratio of grain length to width(f) and Chalkiness degree (g). Different lower-case letters indicate a significant difference $(P<0.05)$ as determined by Duncan's multiple comparasion. $\mu$ : mean value of according trait; ai: additive effect for each QTL controlling according trait. I: epistatic effect between QTLs. P: P-value in SSSL indicated between SSSL and Xihui18 for t-test; P-value in DSSL indicated 
between (DSSLij+ Xihui18) and (SSSLi+ SSSLj) for t-test. S1:(Chr.3 RM3417-RM3766-RM14809RM7425); S2(Chr.3 RM5813--RM3346-RM1221); S3(Chr.5 RM2010-RM405-RM5874); S4(Chr.5 RM2010RM405-RM5874-RM3322--RM3328); S5(Chr.5 RM405--RM5874-RM3322--RM3328); S6(Chr.11 RM7173RM1812--RM6085 ); D1(Chr.3 RM3417--RM3766-RM14809-RM7425, Chr.3 RM5813--RM3346--RM1221); D2(Chr.3 RM3417-RM3766-RM14809-RM7425, Chr.11 RM7173-RM1812-RM6085); The internal markers connected with hyphens indicate the substitution segment from donor, whereas the markers at each end of the substitution segment linked with '--' indicate whether segment recombination might occur.

a

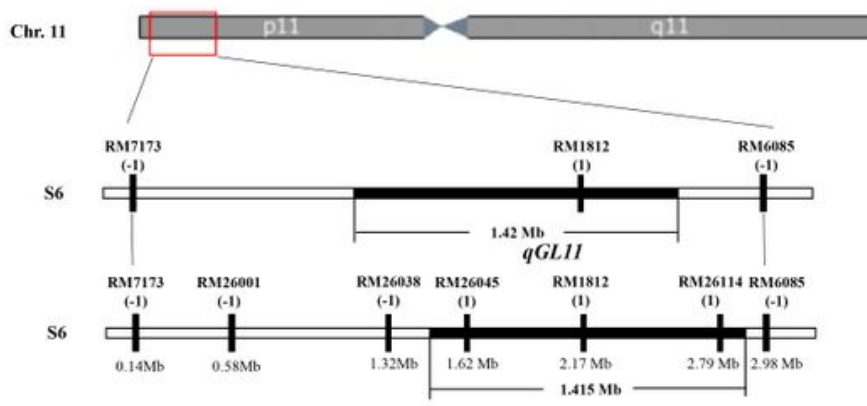

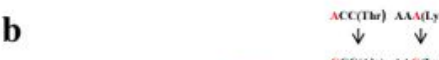

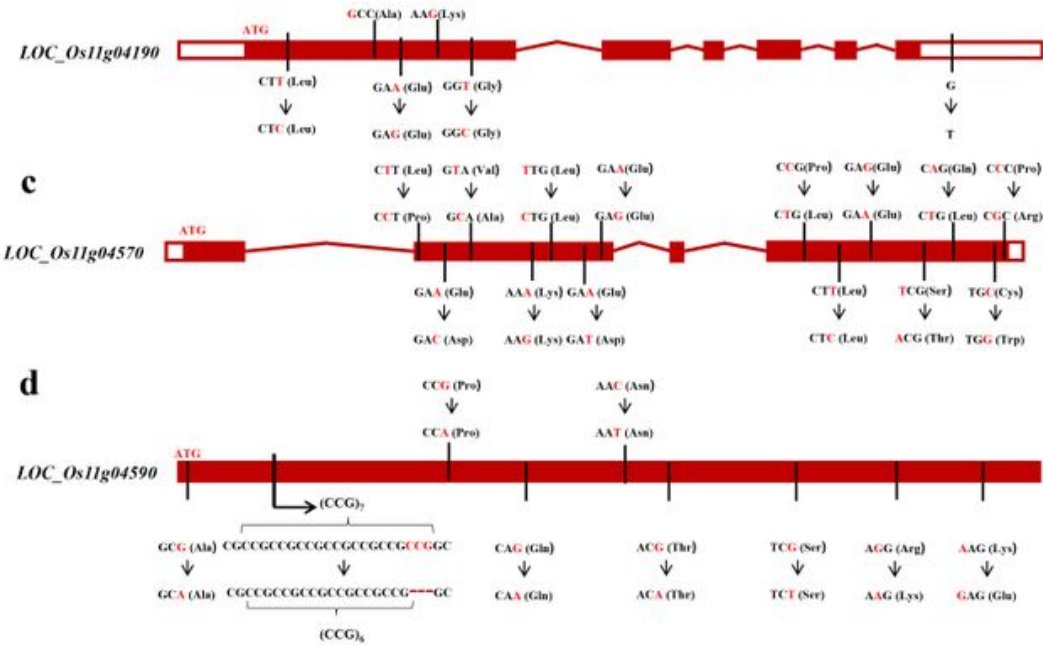

e
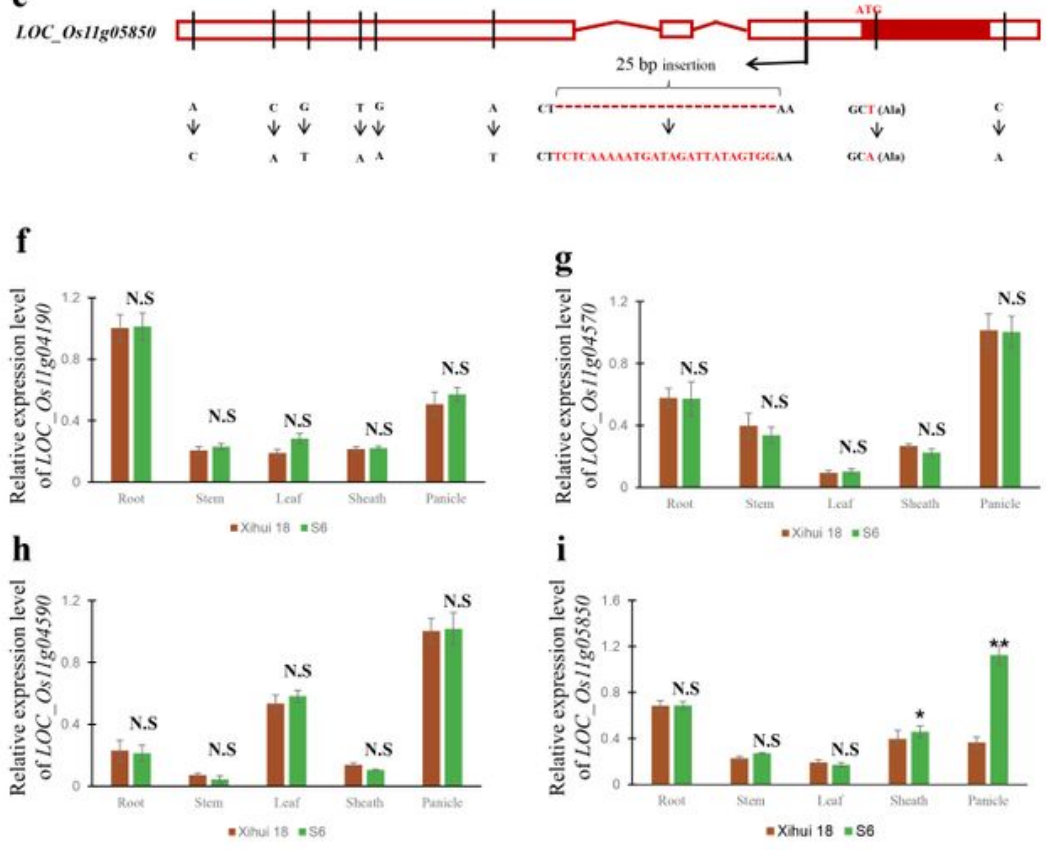


\section{Figure 5}

Sequence analysis and relative expression level of candidate genes for qGL11 between Xihui 18 and S6. a QTL mapping of qGL11. Black regions indicate the estimated length of substitution segment. b-e The DNA sequence of LOC_Os11g04190 (b), LOC_Os11g04570 (c), LOC_Os11g04590 (d) and CycT1;3 (e) in S6 compared with Xihui 18. In candidate gene sequence, the red box represents the coding sequences region, the white box represents $5^{\prime} U T R$ and $3^{\prime} U T R$, the solid red line represents introns, the black line in the gene sequence presents the mutation site, and arrow represents sequence change from Xihui1 8 to S6. f-i Relative expression level of candidate genes LOC_Os11g04190 (f), LOC_Os11g04570 (g), LOC_Os11g04590 (h) and CycT1;3 (i) in root, stem, leaf, sheath and panicle between Xihui 18 and S6. 
a

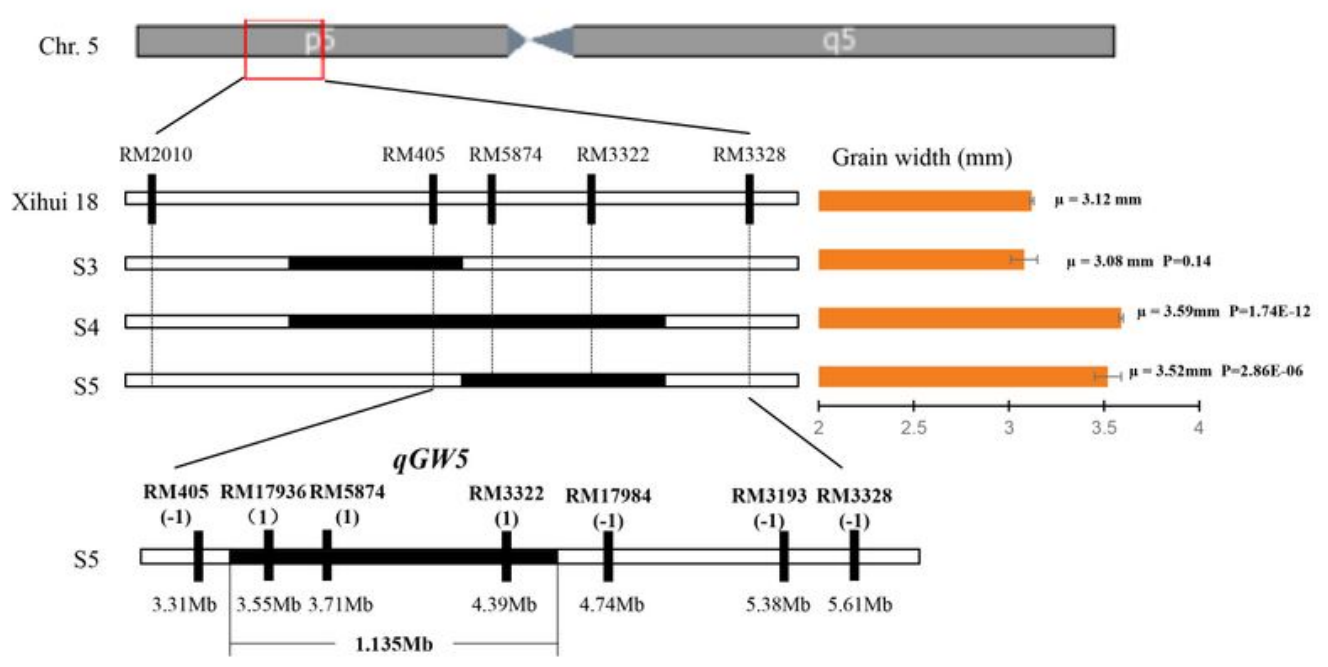

b
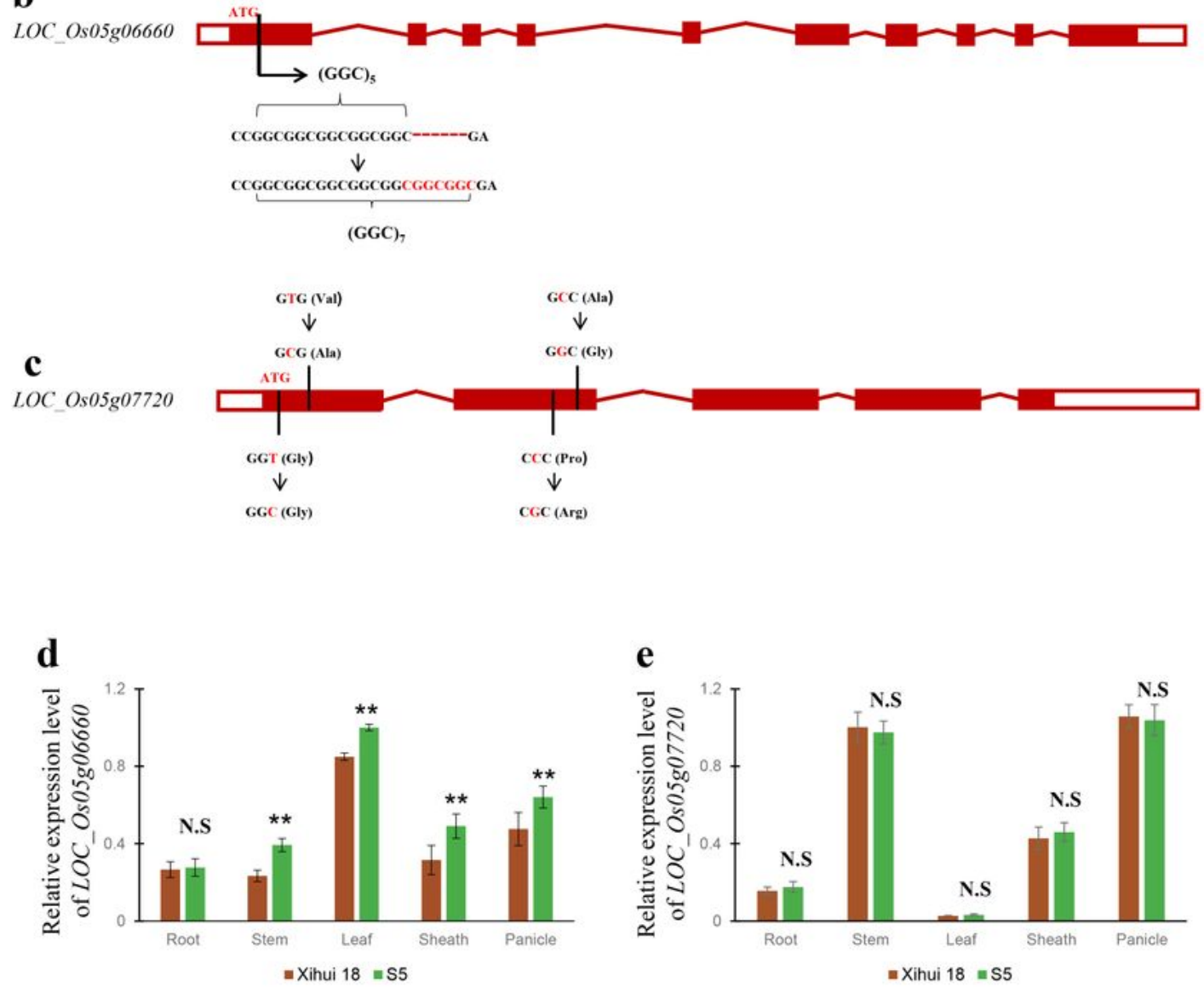

\section{Figure 6}

Sequence analysis and relative expression level of candidate genes for qGW5 between Xihui 18 and S5. a QTL mapping of qGW5. Black regions indicate the estimated length of substitution segment. b-c The DNA sequence of GS5 (b) and OsTAR1 (c) in S5 compared with Xihui 18. In candidate gene sequence, the red box represents the coding sequences region, the white box represents 5'UTR and 3'UTR, the solid red line represents introns, the black line in the gene sequence presents the mutation site, and arrow represents 
sequence change from Xihui18 to S5. d-e Relative expression level of candidate genes GS5 (d) and OsTAR1 (e) in root, stem, leaf, sheath and panicle between Xihui 18 and S5. 